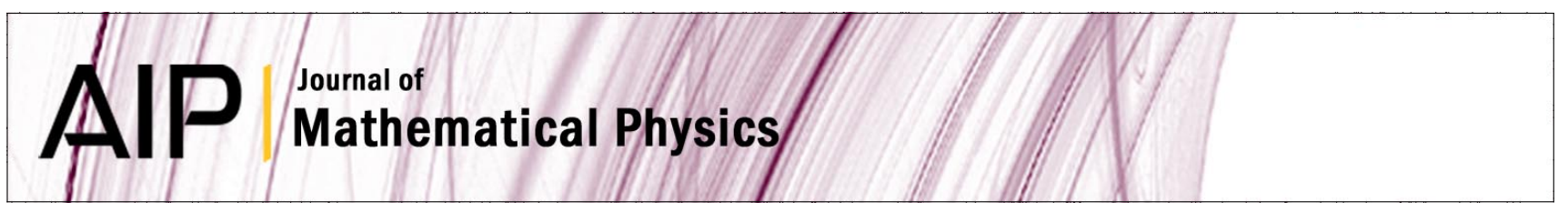

\title{
Covariant constitutive relations and relativistic inhomogeneous plasmas
}

J. Gratus and R. W. Tucker

Citation: J. Math. Phys. 52, 042901 (2011); doi: 10.1063/1.3562929

View online: http://dx.doi.org/10.1063/1.3562929

View Table of Contents: http://jmp.aip.org/resource/1/JMAPAQ/v52/i4

Published by the American Institute of Physics.

\section{Related Articles}

High efficiency coaxial klystron-like relativistic backward wave oscillator with a premodulation cavity Phys. Plasmas 18, 113102 (2011)

Anomalous skin effects in relativistic parallel propagating weakly magnetized electron plasma waves Phys. Plasmas 18, 102115 (2011)

Effects of trapping and finite temperature in a relativistic degenerate plasma

Phys. Plasmas 18, 102306 (2011)

On the bounce-averaging of scattering rates and the calculation of bounce period

Phys. Plasmas 18, 092904 (2011)

Relativistic effects in the interaction of high intensity ultra-short laser pulse with collisional underdense plasma Phys. Plasmas 18, 093108 (2011)

\section{Additional information on J. Math. Phys.}

Journal Homepage: http://jmp.aip.org/

Journal Information: http://jmp.aip.org/about/about_the_journal

Top downloads: http://jmp.aip.org/features/most_downloaded

Information for Authors: http://jmp.aip.org/authors

\section{ADVERTISEMENT}

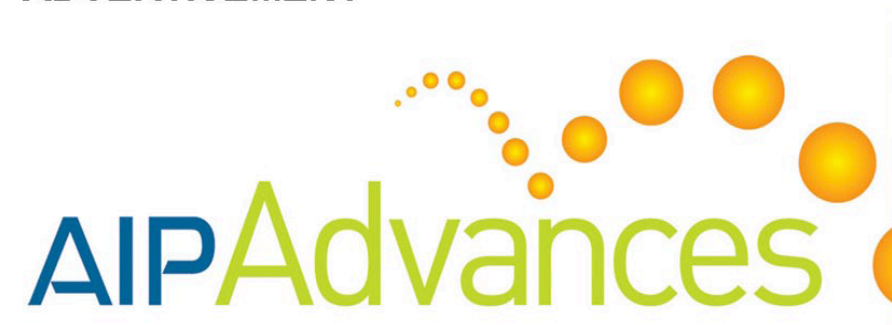

Submit Now

Explore AIP's new

open-access journal

Article-level metrics now available

Join the conversation!

Rate \& comment on articles 


\title{
Covariant constitutive relations and relativistic inhomogeneous plasmas
}

\author{
J. Gratus a) and R. W. Tucker \\ Physics Department, Lancaster University, Lancaster, LA1 4YB, United Kingdom, and \\ The Cockcroft Institute, Daresbury Laboratory, Daresbury Science \& Innovation Campus, \\ Keckwick Lane, Daresbury, Warrington, Cheshire WA4 4AD United Kingdom
}

(Received 24 November 2010; accepted 15 February 2011; published online 4 April 2011)

\begin{abstract}
The notion of a 2-point susceptibility kernel used to describe linear electromagnetic responses of dispersive continuous media in nonrelativistic phenomena is generalized to accommodate the constraints required of a causal formulation in spacetimes with background gravitational fields. In particular the concepts of spatial material inhomogeneity and temporal nonstationarity are formulated within a fully covariant spacetime framework. This framework is illustrated by recasting the Maxwell-Vlasov equations for a collisionless plasma in a form that exposes a 2-point electromagnetic susceptibility kernel in spacetime. This permits the establishment of a perturbative scheme for nonstationary inhomogeneous plasma configurations. Explicit formulae for the perturbed kernel are derived in both the presence and absence of gravitation using the general solution to the relativistic equations of motion of the plasma constituents. In the absence of gravitation this permits an analysis of collisionless damping in terms of a system of integral equations that reduce to standard Landau damping of Langmuir modes when the perturbation refers to a homogeneous stationary plasma configuration. It is concluded that constitutive modeling in terms of a 2-point susceptibility kernel in a covariant spacetime framework offers a natural extension of standard nonrelativistic descriptions of simple media and that its use for describing linear responses of more general dispersive media has wide applicability in relativistic plasma modeling. (c) 2011 American Institute of Physics. [doi:10.1063/1.3562929]
\end{abstract}

\section{INTRODCTION}

The behavior of a material medium in response to electromagnetic and gravitational fields encompasses a vast range of classical and quantum physics. For media composed of a large collection of molecular or ionized structures recourse to a statistical description is required and this often leads to a coarser description in terms of a few thermodynamic variables and their correlations. Such a description relies on the efficacy of particular constitutive models or phenomenological constitutive data that serve to circumscribe its domain of applicability.

For phenomena where the relative motions of the constituents approach the speed of light in vасио or the material experiences bulk accelerations or gravitational interactions such constitutive descriptions must be formulated within a relativistic framework. However, even within a spacetime covariant formulation there remains great freedom in how to accommodate electromagnetic responses that depend on material dispersion induced by spatial correlations or temporal delays of electromagnetic interactions. ${ }^{1}$ The incorporation of such effects in a theoretical description often relies on a detailed structural model of the medium particularly if it is inhomogeneous or external gravitational gradients are relevant. Notwithstanding these complexities simple constitutive models have proved of considerable value for homogeneous polarizable media that exhibit temporal

a) Author to whom correspondence should be addressed. Electronic mail: j.gratus@lancaster.ac.uk. 
dispersion in a laboratory frame where gravity plays no essential role. Indeed the notion of permittivity and permeability tensors is often adequate to parameterize a large range of experimental linear responses of simple polarizable media to external static and dynamic electromagnetic fields. More generally, for nondispersive media these tensors can be subsumed into a susceptibility kernel that readily accommodates special relativistic effects on the bulk motion of media.

In this article the degree to which the notion of a susceptibility kernel can be generalized to describe linear electromagnetic responses of dispersive continuous media is explored. In particular the effects of spatial material inhomogeneity and nonstationarity will be formulated within a fully covariant spacetime framework. In this manner the formulation can accommodate arbitrary gravitational and electromagnetic interactions. The framework will be illustrated by recasting the Maxwell-Vlasov equations for a collisionless plasma in a form that exposes a 2-point ${ }^{2}$ electromagnetic susceptibility kernel in an arbitrary external gravitational field. This permits the establishment of a perturbative scheme for nonstationary inhomogeneous plasma configurations in terms of such a kernel. Explicit formulae for the perturbed kernel are derived in both the presence and absence of gravitation in terms of the general solution to the equations of motion of the plasma constituents. In the absence of gravitation this permits an analysis of collisionless damping in terms of a system of integral equations that reduce to standard Landau damping of Langmuir modes when the perturbation refers to a homogeneous stationary plasma configuration.

It is concluded that constitutive modeling in terms of a 2-point susceptibility kernel in a covariant spacetime framework offers a natural extension of standard nonrelativistic descriptions of simple media and that its use for describing linear responses of more general dispersive media has wide applicability in relativistic plasma modeling.

\section{CONSTITUTIVE RELATIONS}

In the following spacetime $M$ is considered a globally hyperbolic, topologically trivial fourdimensional manifold endowed with a metric tensor $g$ with signature $(-1,+1,+1,+1)$ describing gravitation. A closed 2-form $F$ describes the electromagnetic field. The bundle of exterior $p$-forms over $M$ is denoted $\Lambda^{p} M$ and its sections $\Gamma \Lambda^{p} M$ are $p$-forms on $M$. The bundle of all forms is $\Lambda M=\bigcup_{p=0}^{p=4} \Lambda^{p} M$. Associated with $g$ is the Hodge map $\star$. Thus for $\alpha \in \Gamma \Lambda^{p} M$ its corresponding Hodge dual is denoted $\star \alpha \in \Gamma^{4-p} \Lambda M$. The tangent bundle over $M$ is denoted $T M$ and its sections $\Gamma T M$ are vector fields on $M$. We call the 1 -form $\widetilde{J}=g(J,-) \in \Gamma \Lambda^{1} M$ the metric dual of the vector field $J \in \Gamma T M$. Maxwell's equations for the electromagnetic field $F \in \Gamma \Lambda^{2} M$ in a polarizable medium containing an electric current $J \in \Gamma T M$, satisfying the continuity (or current conservation) equation $d \star \widetilde{J}=0$, are written

$$
d F=0, \quad d \star G=-\star \widetilde{J} .
$$

The excitation 2-form $G \in \Gamma \Lambda^{2} M$ can always be expressed,

$$
G=\epsilon_{0} F+\Pi,
$$

in terms of the permittivity $\epsilon_{0}$ of free space. The polarization ${ }^{3}$ 2-form $\Pi \in \Gamma \Lambda^{2} M$ results from all electromagnetic field sources not made explicit in $J$.

In general $\Pi$ and $J$ are nonlinear functionals of $F$ and other fields such as matter and initial data on any initial spacelike hypersurface $\Sigma_{M} \subset M$. Such functionals are the constitutive relations describing $G$ and $J$ in terms of $F$ and the other fields.

It is convenient to introduce integration on a fibered manifold $\mathcal{N}$ of dimension $n+r$ with projection $\pi_{\mathcal{N}}: \mathcal{N} \rightarrow N$ over a manifold $N$ of dimension $n$. Thus at each point $\sigma \in N$ one has the fiber $\mathcal{N}_{\sigma}=\pi_{\mathcal{N}}^{-1}\{\sigma\}=\left\{\left(\sigma^{\prime}, \zeta\right) \in \mathcal{N} \mid \pi_{\mathcal{N}}\left(\sigma^{\prime}, \zeta\right)=\sigma\right\}$ so $\operatorname{dim}\left(\mathcal{N}_{\sigma}\right)=r$ is the fiber dimension. For $\alpha \in \Gamma \Lambda^{p+r} \mathcal{N}$ we define ${ }^{4,5}$ the form $\mathscr{\oiint}_{\pi_{\mathcal{N}}} \alpha \in \Gamma \Lambda^{p} N$ by

$$
\int_{N} \beta \wedge \oint_{\pi_{\mathcal{N}}} \alpha=\int_{\mathcal{N}} \pi_{\mathcal{N}}^{\star}(\beta) \wedge \alpha
$$

for all $\beta \in \Gamma \Lambda^{n-p} N$. 
In terms of local coordinates $\left(\sigma^{1}, \ldots, \sigma^{n}\right)$ and $\left(\sigma^{1}, \ldots, \sigma^{n}, \varsigma^{1} \ldots \varsigma^{r}\right)$ for patches on $N$ and $\mathcal{N}$, respectively, one may write the fiber integral

$$
\left.\left(\oint_{\pi_{\mathcal{N}}} \alpha\right)\right|_{\sigma}=\left.\sum_{1 \leq I_{1}<\ldots<I_{p} \leq n} d \sigma^{I_{1}} \wedge \ldots \wedge d \sigma^{I_{p}} \int_{\zeta \in \mathcal{N}_{\sigma}} i_{\partial / \partial \sigma^{I_{p}}} \ldots i_{\partial / \partial \sigma^{I_{1}}} \alpha\right|_{(\sigma, \zeta)}
$$

where $\mathcal{N}_{\sigma}=\pi_{\mathcal{N}}^{-1}(\{\sigma\})$ is the fiber over the point $\sigma \in N$ and $i_{\partial / \partial \sigma I_{k}}$ is the contraction on forms. Observe that if $\alpha$ does not contain the factor $d \varsigma^{1} \wedge \cdots \wedge d \varsigma^{r}$ then $\oint_{\pi_{\mathcal{N}}} \alpha=0$. The proof of this is given in Lemma 2 in the Appendix.

A key result of fiber integration, used to establish the current continuity equation, is that it commutes with the exterior derivative,

$$
\left.\left(d \oint_{\pi_{\mathcal{N}}} \alpha\right)\right|_{\sigma}=\left.\left(\oint_{\pi_{\mathcal{N}}} d \alpha\right)\right|_{\sigma}
$$

for $\sigma$ not on the boundary of $N$ provided the support of $\alpha$ does not intersect the boundary of $\mathcal{N}$. The proof is given in Lemma 3 in the Appendix.

In general models for $\Pi$ demand a knowledge of the dynamics of sources responsible for polarization as well as any permanent polarization that may exist in the medium. A full dynamical description depends on a specification of appropriate initial value data $\zeta$ on $\Sigma_{M}$. The exact structure of $\zeta$ depends on the sources of the polarization. For the plasma model described in Sec. III the initial data corresponds to the velocity profile for each particle species at each point on $\Sigma_{M}$ in the plasma.

In this article $\Pi$ is considered to be an affine functional of $F$ of the form

$$
\Pi[F, \zeta]=\oint_{p_{X}} \chi \wedge p_{Y}^{\star}(F)+Z[\zeta]
$$

for some functional $Z$ of $\zeta$. The first term on the right is expressed in terms of the fiber integral of a 2-point susceptibility kernel $\chi \in \Gamma \Lambda^{4}\left(M_{X} \times M_{Y}\right)$ expressible locally as

$$
\chi=\frac{1}{4} \chi_{a b c d}(x, y) d x^{a} \wedge d x^{b} \wedge d y^{c} \wedge d y^{d} .
$$

Here $M_{X}$ and $M_{Y}$ are two copies of $M$, locally coordinated by $\left(x^{0}, \ldots, x^{3}\right)$ and $\left(y^{0}, \ldots, y^{3}\right)$, respectively, with projections $p_{X}: M_{X} \times M_{Y} \rightarrow M_{X}, p_{Y}: M_{X} \times M_{Y} \rightarrow M_{Y}, p_{X}(x, y)=x, p_{Y}(x, y)$ $=y$ and initial hypersurfaces $\Sigma_{M_{X}} \subset M_{X}$ and $\Sigma_{M_{Y}} \subset M_{Y}$. Throughout, summation is over roman indices $a, b, c=0,1,2,3$ and greek indices $\mu, \nu, \sigma=1,2,3$.

To consistently remove any reference to $M$ (without a subscript) let $F \in \Gamma \Lambda^{2} M_{Y}, \epsilon_{0} F \in$ $\Gamma \Lambda^{2} M_{X}, G \in \Gamma \Lambda^{2} M_{X}, J \in \Gamma T M_{X}$, and $\Pi[F, \zeta] \in \Gamma \Lambda^{2} M_{X}$. Thus $\epsilon_{0}$ can be regarded as a map $\epsilon_{0}: \Gamma \Lambda^{2} M_{Y} \rightarrow \Gamma \Lambda^{2} M_{X}$ which is the pullback of the natural isomorphism $M_{X} \rightarrow M_{Y}$, together with a scaling to accommodate the choice of electromagnetic units.

In terms of local coordinate bases on $M_{X}$ and $M_{Y}$ the components of (6) are

$$
\Pi[F, \zeta]_{a b}(x)=\int_{y \in M} \chi_{a b c d}(x, y) F_{e f}(y) d y^{c d e f}+Z[\zeta]_{a b}
$$

in a multiindex notation with

$$
\begin{gathered}
d x^{a_{1} \ldots a_{p}} \equiv d x^{a_{1}} \wedge \cdots \wedge d x^{a_{p}}, \\
i_{a_{1} \ldots a_{p}}^{(x)_{p}} \equiv i_{\frac{\partial}{\partial x^{a_{p}}}} \cdots i_{\frac{\partial}{\partial x^{a_{1}}}} .
\end{gathered}
$$

(Note the reverse order for internal contraction.) Summations over multiindices $I \subset\{1, \ldots, n\}$ considered as an ordered $p$-list $I_{1}<I_{2}<\ldots<I_{p}$ of length $|I|=p$ will also be employed. Thus

$$
\begin{gathered}
d x^{I} \equiv d x^{I_{1} \cdots I_{p}}=d x^{I_{1}} \wedge \cdots \wedge d x^{I_{p}}, \\
i_{I}^{(x)} \equiv i_{I_{1} \cdots I_{p}}^{(x)}=i_{\frac{\partial}{\partial x^{I} p}} \cdots i_{\frac{\partial}{\partial x^{I_{1}}}}
\end{gathered}
$$

so that, via summation, if $\alpha \in \Gamma \Lambda^{p} M$ then $d x^{I} \wedge i_{I}^{(x)} \alpha=\alpha$, where $|I|=p$. 
In this notation the product manifold $M_{X} \times M_{Y}$ inherits the following maps that will be employed below

$$
\begin{gathered}
d_{X}: \Gamma \Lambda^{p}\left(M_{X} \times M_{Y}\right) \rightarrow \Gamma \Lambda^{p+1}\left(M_{X} \times M_{Y}\right), \\
d_{X}(\alpha)=\frac{\partial \alpha_{I J}}{\partial x^{a}} d x^{a} \wedge d x^{I} \wedge d y^{J} \\
d_{Y}: \Gamma \Lambda^{p}\left(M_{X} \times M_{Y}\right) \rightarrow \Gamma \Lambda^{p+1}\left(M_{X} \times M_{Y}\right), \\
d_{Y}(\alpha)=\frac{\partial \alpha_{I J}}{\partial y^{a}} d y^{a} \wedge d x^{I} \wedge d y^{J} \\
\star_{X}: \Gamma \Lambda\left(M_{X} \times M_{Y}\right) \rightarrow \Gamma \Lambda\left(M_{X} \times M_{Y}\right), \\
\star_{X}(\alpha)=\alpha_{I J}\left(\star d x^{I}\right) \wedge d y^{J},
\end{gathered}
$$

where $\alpha=\alpha_{I J} d x^{I} \wedge d y^{J}$.

Since $F=d A$ and for $A$ with compact support away from any boundary of $M_{Y}$ it follows from (6) that

$$
\Pi[F, \zeta]=-\oint_{p_{X}}\left(d_{Y} \chi\right) \wedge p_{Y}^{\star}(A)+Z[\zeta]
$$

Hence $\Pi[F, \zeta]$ remains invariant ${ }^{6}$ under the gauge transformation

$$
\chi \longrightarrow \chi+d_{Y} \check{\zeta}
$$

for any $\check{\zeta}=\check{\zeta}_{a b c} d x^{a b} \wedge d y^{c} \in \Gamma \Lambda^{3}\left(M_{X} \times M_{Y}\right)$. Since the support of $A$ can be made arbitrarily small $d_{Y} \chi$ is uniquely specified by $\Pi[F, \zeta]$. Furthermore,

$$
d \star \Pi[F, \zeta]=-\oint_{p_{X}}\left(d_{X} \star_{X} d_{Y} \chi\right) \wedge p_{Y}^{\star}(A)+d \star Z[\zeta],
$$

hence $d \star \Pi[F, \zeta]$ is invariant under the gauge transformation

$$
\chi \longrightarrow \chi+d_{Y} \check{\zeta}+\star_{X} d_{X} \check{\xi}
$$

for any $\check{\zeta}=\check{\zeta}_{a b c} d x^{a b} \wedge d y^{c}$ and $\check{\xi}=\check{\xi}_{a b c} d x^{a} \wedge d y^{b c}$. Similarly $d_{X} \star_{X} d_{Y} \chi$ is uniquely determined by $d \star \Pi[F, \zeta]$.

In general, the permittivity functional $\Pi$ is a nonlocal functional in spacetime given by the integral (8). If $\chi$ is smooth, and not identically zero, then $\Pi$ is always nonlocal. However, for distributional susceptibility kernels it is possible for $\Pi$ to remain local. In this category one has the local, linear Minkowski constitutive relations

$$
\Pi[F]=\epsilon_{0}\left(\epsilon_{r}-1\right) i_{v} F \wedge \tilde{v}+\epsilon_{0}\left(\mu_{r}^{-1}-1\right) \star\left(\left(i_{v} \star F\right) \wedge F\right),
$$

where $v \in \Gamma T M_{Y}$ is a vector field representing the bulk 4-velocity of the medium and $\epsilon_{r}, \mu_{r} \in$ $\Gamma \Lambda^{0} M_{Y}$ are the relative permittivity and permeability scalars of the medium. These relations can be represented by a distributional susceptibility kernel with support on the diagonal set $\left\{(x, y) \in M_{X} \times M_{Y} \mid x=y\right\}$.

In general $\Pi$ is said to be causal on all of $M$ if $\left.\Pi\right|_{x}$ only depends of the values of $F$ which lie on or within the past light-cone ${ }^{7,8} J^{-}(x) \subset M_{Y}$ of $x$. If $\Pi$ depends on $\zeta$ it may be causal on $M_{X}^{+}$, where $M_{X}^{+}=\Sigma_{M_{X}} \cup\left\{x\right.$ lies to the future of $\left.\Sigma_{M_{X}}\right\}$. The functional $\Pi$ is causal on $M_{X}^{+}$if $\left.\Pi[F, \zeta]\right|_{x}$ only depends on the values of $F$ and $\zeta$ which lie on or within its past light-cone $J^{-}(x) \cap M_{X}^{+}$of $x$ and $x \in M_{X}^{+}$. The data functional $Z$ is casual on $M_{X}^{+}$if $\left.Z[\zeta]\right|_{x}$ depends only on $\zeta \in \Sigma_{M_{X}} \cap J^{-}(x)$ for all $x \in M_{X}^{+}$. For $\Pi$ to be causal on $M_{X}^{+}$it is necessary and sufficient (Lemma 5 in the Appendix) that the following be satisfied:

- $Z$ is causal on $M_{X}^{+}$,

- $\left.\left(d_{Y} \chi\right)\right|_{(x, y)}=0$ for all $(x, y) \in M_{X}^{+} \times M_{Y}^{+}$such that $y \notin J^{-}(x)$, and

- $\left.\iota_{\Sigma_{M_{Y}}}^{\star}(\chi)\right|_{(x, y)}=0$ for all $(x, y) \in M_{X}^{+} \times \Sigma_{M_{Y}}$ such that $y \notin J^{-}(x)$, where $\iota_{\Sigma_{M_{Y}}}: M_{X}^{+} \times$ $\Sigma_{M_{Y}} \hookrightarrow M_{X}^{+} \times M_{Y}^{+}$is the natural embedding. 


\section{A. Spacetime homogeneous constitutive relations for media in Minkowski spacetime}

Minkowski spacetime has properties that underpin the notions of material spatial homogeneity and stationary processes. Being isomorphic to a real four-dimensional vector space it can be given an affine structure in addition to its light-cone structure. Physically this implies that no particular point in a spacetime without gravitation has a distinguished status and the concepts of material and field energy, momentum, and angular momentum can be defined in terms of the Killing symmetries of the spacetime metric. Since all points of the spacetime are equivalent relative to this affine structure it is sufficient to denote $M_{X}$ and $M_{Y}$ by $M$ and, relative to any point chosen as origin, a point with coordinates $x$ can be identified with a vector denoted by $x \in \mathbb{R}^{4}$. It is then convenient to introduce the Minkowski translation map $A_{z}: M \rightarrow M, A_{z}(x)=x+z$ that maps points $x$ to $x+z$ on $M$.

If the electromagnetic properties of an unbounded medium are independent of location in spacetime they will be called spacetime homogeneous. Such electromagnetic constitutive properties imply that variations in $F$ at event $y \in M$ produce an induced variation in a functional $\Pi_{\mathbf{H}}[F]$ at event $x \in M$, via a kernel $\chi_{a b c d}(x, y)$ that depends on the 4 -vector $x-y$. If the constitutive relation is causal then there is no induced variation if $x \notin J^{+}(y)$. Furthermore, in a spacetime homogeneous medium $Z[\zeta]=Z_{\mathbf{H}}$, where $Z_{\mathbf{H}} \in \Gamma \Lambda^{2} M$ is independent of $\zeta$.

In terms of $A_{z}$ an electromagnetic constitutive functional $\Pi_{\mathbf{H}}$ is given by

$$
\Pi_{\mathbf{H}}[F]=\oint_{p_{X}} \chi \wedge p_{Y}^{\star}(F)+Z_{\mathbf{H}}
$$

The functional $\Pi_{\mathbf{H}}$ is said to be spacetime homogeneous ${ }^{9}$ if

$$
\Pi_{\mathbf{H}}\left[A_{z}^{\star} F\right]=A_{z}^{\star} \Pi_{\mathbf{H}^{[}}[F] .
$$

This follows if the susceptibility kernel $\chi$ satisfies

$$
\left.\chi\right|_{(x+z, y+z)}=\left.\chi\right|_{(x, y)}
$$

and $A_{Z}^{\star} Z_{\mathbf{H}}=Z_{\mathbf{H}}$. The contribution $Z_{\mathbf{H}}$ may model the presence of an externally prescribed stationary uniform permanent magnetic or electric polarization. Equation (13) implies the components of $\chi$ in (7) can be written

$$
\chi_{a b c d}(x, y)=X_{a b c d}(x-y),
$$

where

$$
X_{a b c d}(x)=\chi_{a b c d}(x, 0) .
$$

Thus, in a Minkowski spacetime for materials with electromagnetic spacetime homogeneous properties, (8) can be written in terms of a convolution integral

$$
\begin{aligned}
\Pi_{\mathbf{H}}[F]_{a b}(x) & =\frac{1}{4} \int_{y \in M} X_{a b c d}(x-y) F_{e f}(y) d y^{c d e f}+\left(Z_{\mathbf{H}}\right)_{a b} \\
& \equiv \frac{1}{4} \epsilon^{c d e f}\left(X_{a b c d} * F_{e f}\right)(x)+\left(Z_{\mathbf{H}}\right)_{a b},
\end{aligned}
$$

where $\epsilon^{\text {cdef }}= \pm 1,0$ denotes the Levi-Civita alternating symbol in coordinates in which the metric tensor takes the form $g=\eta_{a b} d x^{a} \otimes d x^{b}$, where $\eta_{a b}=\operatorname{diag}(-1,+1,+1,+1)$. In these coordinates the $\left(Z_{\mathbf{H}}\right)_{a b}$ are all constants.

Let $\hat{F}_{e f}(k)$ and $\hat{\Pi}_{\mathbf{H}}[F]_{a b}(k)$ denote the Fourier transforms of $F_{e f}(x)$ and $\Pi_{\mathbf{H}}[F]_{a b}(x)$, respectively, i.e.,

$$
\begin{gathered}
\hat{F}_{e f}(k)=\int_{x \in \mathbb{R}^{4}} F_{e f}(x) e^{i k \cdot x} d x^{0123}, \\
\hat{\Pi}_{\mathbf{H}}[F]_{a b}(k)=\int_{x \in \mathbb{R}^{4}} \Pi_{\mathbf{H}^{[}[F]_{a b}(x) e^{i k \cdot x} d x^{0123},}
\end{gathered}
$$


where $k=k_{a} d x^{a}, k \cdot x=k_{a} x^{a}$. Similarly let $\hat{X}_{a b}{ }^{e f}(k)$ be the Fourier transformation of $\frac{1}{2} \epsilon^{\text {cdef }} X_{a b c d}(x)$, i.e.,

$$
\hat{X}_{a b}{ }^{e f}(k)=\frac{1}{2} \epsilon^{c d e f} \int_{x \in \mathbb{R}^{4}} X_{a b c d}(x) e^{i k \cdot x} d x^{0123} .
$$

If $Z_{\mathbf{H}}=0$ then it follows from (16) that

$$
\hat{\Pi}_{\mathbf{H}}[F]_{a b}(k)=\frac{1}{2} \hat{X}_{a b}{ }^{c d}(k) \hat{F}_{c d}(k) .
$$

Since $\chi_{a b c d}$ is a real function on $M$ its Fourier transform satisfies

$$
\hat{X}_{a b}{ }^{c d}(k)^{*}=\hat{X}_{a b}{ }^{c d}(-k) .
$$

The 36 components of $\hat{X}_{a b}{ }^{c d}(k)$ subject to this symmetry can be expressed in terms of permittivity, permeability, and magnetoelectric tensors relative to any observer frame. A specification of these components together with relations that determine the electric current $J$ serve as an electromagnetic model for a spacetime homogeneous medium in Minkowski spacetime. If the medium lacks this electromagnetic homogeneity recourse to the Fourier transform (16) is not possible and the constitutive properties must be given in terms of a 2-point kernel and (8).

\section{CONSTITUTIVE MODELS FOR A COLLISIONLESS IONIZED PLASMA}

As noted in the Introduction the computation of the susceptibility for homogeneous stationary dispersive media owes much to phenomenological models and input from experiment. For certain conductors, semiconductors, insulators, and low-dimensional structures much can also be learnt from the application of quantum theory. For inhomogeneous and anisotropic media subject to nonstationary electromagnetic fields linear responses are often the subject of a perturbation approach. This is particularly so in the case of ionized gases.

As an application of the above formalism the classical linear response of a fully ionized inhomogeneous nonstationary collisionless plasma to a perturbation is considered in the presence of an arbitrary background gravitational field. The perturbed constitutive tensor will be calculated in terms of solutions to the classical Maxwell-Vlasov equations for the system. This system is described in terms of the electromagnetic 2-form $F \in \Gamma \Lambda^{2} M^{+}$over a gravitational spacetime $M^{+}$, lying in the future of an initial hypersurface $\Sigma_{M}$, and a collection of "one-particle distribution" forms (of degree 6), $\theta^{\lfloor\alpha\rceil} \in \Gamma \Lambda^{6} \mathcal{E}^{+}$(one for each charged species of particle $\lfloor\alpha\rceil$ with mass $m^{\lfloor\alpha\rceil}$ and charge $q^{\lfloor\alpha\rceil}$ ) on the upper unit hyperboloid bundle $\pi: \mathcal{E}^{+} \rightarrow M^{+}$over $M^{+}$. The seven-dimensional manifold $\mathcal{E}^{+}$is a subbundle of the eight-dimensional tangent bundle $T M^{+}$over $M^{+}$whose sections are all future pointing timelike unit vector fields on $M^{+}$. Thus generic elements of $\mathcal{E}^{+}$can be written $(z, w)$ with $z \in M^{+}, \pi(z, w)=z$, and $g(w, w)=-1$. The initial values of the one-particle forms are given on the hypersurface $\Sigma_{\mathcal{E}}$, where $\Sigma_{\mathcal{E}}=\pi^{-1}\left\{\Sigma_{M}\right\} \subset \mathcal{E}^{+}$.

The Maxwell-Vlasov system is usually written in terms of the Maxwell system in vacuo and all sources are contained in the total current $J \in \Gamma T M^{+}$. This in turn is given by the sum over each species current

$$
J=\sum_{\lfloor\alpha\rceil} J^{\lfloor\alpha\rceil}
$$

where $J^{\lfloor\alpha\rceil} \in \Gamma T M^{+}$. Thus in terms of $F$ and $J$ the Maxwell subsystem is

$$
d F=0, \epsilon_{0} d \star F=-\star \widetilde{J} .
$$

The dynamic equations for each $\theta^{\mid \alpha\rceil}$ can be written succinctly in terms of forms on $\mathcal{E}^{+}$and a collection of Liouville vector fields $W^{\lfloor\alpha]} \in \Gamma T \mathcal{E}^{+}$describing the flow of the charged particles associated with each species $[\alpha]$,

$$
\left.W^{\lfloor\alpha\rceil}\right|_{(z, w)}=\mathcal{H}_{(z, w)}(z, w)+\frac{q^{\lfloor\alpha\rceil}}{m^{\lfloor\alpha\rceil}} \mathcal{V}_{(z, w)}\left(\widetilde{i_{(z, w)} F}\right)
$$


in terms of certain horizontal and vertical lifts. ${ }^{10}$ With these vector fields the distribution forms $\theta$ ${ }^{\lfloor\alpha\rceil}$ are defined to satisfy the collisionless conditions,

$$
\begin{gathered}
d \theta^{\lfloor\alpha\rceil}=0, \\
i_{W^{\lfloor\alpha]}} \theta^{\lfloor\alpha]}=0 .
\end{gathered}
$$

To close this system one requires

$$
\star \widetilde{J^{\lfloor\alpha\rceil}}=q^{\lfloor\alpha\rceil} \oint_{\pi} \theta^{\lfloor\alpha\rceil} .
$$

The closure of $\theta^{\lfloor\alpha]}$ leads, from (5), to the continuity equation for each species current,

$$
d \star \widetilde{J}^{[\alpha]}=d\left(\oint_{\pi} \theta^{\mid \alpha]}\right)=\oint_{\pi} d \theta^{\mid \alpha\rceil}=0,
$$

so the total current 3 -form $\star \widetilde{J}$ is closed away from the boundary $\Sigma_{M}$.

A local coordinate system $\left(z^{0}, \ldots, z^{3}\right)$ for a region containing $z$ on $M^{+}$induces a local coordinate system $\left(z^{0}, \ldots, z^{3}, w^{1}, w^{2}, w^{3}\right)$ on $\mathcal{E}^{+}$. Since $\mathcal{E}^{+} \subset T M^{+}$the tangent vector for a generic element $(z, w) \in \mathcal{E}^{+}$may be written

$$
(z, w)=\left.w^{a} \frac{\partial}{\partial z^{a}}\right|_{z} \in \mathcal{E}_{z}^{+} \subset T_{z} M^{+},
$$

where $\mathcal{E}_{z}^{+}=\pi^{-1}(\{z\})$ is the three-dimensional fiber of $\mathcal{E}^{+}$over $z$ coordinated by $\left(w^{1}, w^{2}, w^{3}\right)$ and $w^{0}(z, w)$ is the solution to $g_{a b} w^{a} w^{b}=-1$ with $w^{0}>0$. All indices in the range $0,1,2,3$ are raised and lowered using $g^{a b}$ and $g_{a b}$ so that $w_{0}=w^{a} g_{a 0}$. Given a pair of vectors $(z, w),(z, v) \in \mathcal{E}_{z}^{+} \subset$ $T_{z} M^{+}$the horizontal lift of the vector $(z, v)$ to the point $(z, w) \in \mathcal{E}^{+}$will be denoted $\mathcal{H}_{(z, w)}(z, v) \in$ $T_{(z, w)} \mathcal{E}^{+}$and is given by

$$
\mathcal{H}_{(z, w)}(z, v)=\left.\left(v^{a} \frac{\partial}{\partial z^{a}}-\Gamma^{v}{ }_{e f}(z) w^{e} v^{f} \frac{\partial}{\partial w^{v}}\right)\right|_{(z, w)},
$$

where $\Gamma^{a}{ }_{e f}$ are the Christoffel symbols determined by the metric components $g^{a b}$. Furthermore, if $g(v, w)=0$ then the vertical lift of the vector $(z, v)$ to the point $(z, w) \in \mathcal{E}^{+}$is given by

$$
\mathcal{V}_{(z, w)}(z, v)=\left.\left(v^{\mu} \frac{\partial}{\partial w^{\mu}}\right)\right|_{(z, w)} \in T_{(z, w)} \mathcal{E}^{+}
$$

Thus from (21), each Liouville vector field in these coordinates can be expressed as

$$
\left.W^{\lfloor\alpha\rceil}\right|_{(z, w)}=w^{a} \frac{\partial}{\partial z^{a}}+\left(-\Gamma_{e f}^{v}(z) w^{e} w^{f}+\frac{q^{\mid \alpha\rceil}}{m^{\lfloor\alpha\rceil}} F_{e f}(z) g^{\nu e} w^{f}\right) \frac{\partial}{\partial w^{v}}
$$

Denote by $\Omega \in \Gamma \Lambda^{7} \mathcal{E}^{+}$the natural 7-form measure on $\mathcal{E}^{+}$given in these coordinates by

$$
\Omega=\frac{|\operatorname{det} g|}{w_{0}} d z^{0123} \wedge d w^{123} .
$$

In Ref. 11, Eq. (94)] it is shown that for all species $\lfloor\alpha\rceil$,

$$
d i_{W^{\lfloor\rceil}} \Omega=0 .
$$

The distribution function $f^{\lfloor\alpha]} \in \Gamma \Lambda^{0} \mathcal{E}^{+}$relative to $\Omega$ for the species $\lfloor\alpha\rceil$ is defined implicitly via

$$
\theta^{\lfloor\alpha\rceil}=i_{W^{\lfloor\alpha\rceil}}\left(f^{\lfloor\alpha]} \Omega\right)
$$

From (30) and (31) it follows that (23) is equivalent to

$$
W^{\lfloor\alpha\rceil}\left(f^{\lfloor\alpha\rceil}\right)=0
$$

and from (24) the components of the species current $\lfloor\alpha\rceil$ are given in terms of $f^{\lfloor\alpha\rfloor}(z, w)$ by

$$
J^{\lfloor\alpha\rceil b}(z)=q^{\mid \alpha\rceil} \int_{\mathcal{E}_{z}^{+}} \frac{w^{b}|(\operatorname{det} g)(z)|^{1 / 2}}{w_{0}(z, w)} f^{\lfloor\alpha\rceil}(z, w) d w^{123} .
$$




\section{A. Perturbation analysis}

Let $\theta_{1}^{[\alpha]} \in \Gamma \Lambda^{6} \mathcal{E}^{+}$and $F_{1} \in \Gamma \Lambda^{2} M^{+}$be perturbations of $\theta_{0}^{[\alpha]}$ and $F_{0}$, i.e.,

$$
\theta^{\lfloor\alpha\rceil}=\theta_{0}^{\lfloor\alpha\rceil}+\theta_{1}^{\lfloor\alpha\rceil}+\cdots, \quad F=F_{0}+F_{1}+\cdots,
$$

where

$$
\begin{gathered}
d \theta_{0}^{\lfloor\alpha\rceil}=0, \quad i_{W_{0}^{\lfloor\alpha]}} \theta_{0}^{\lfloor\alpha\rceil}=0, \\
d F_{0}=0, \quad \epsilon_{0} d \star F_{0}=-\sum_{\lfloor\alpha\rceil} q^{\lfloor\alpha\rceil} \oint_{\pi} \theta_{0}^{\lfloor\alpha\rceil}, \\
\left.W_{0}^{\lfloor\alpha\rceil}\right|_{(z, w)}=\mathcal{H}_{(z, w)}(z, w)+\frac{q^{\lfloor\alpha\rceil}}{m^{\lfloor\alpha\rceil}} \mathcal{V}_{(z, w)}\left(\widetilde{i_{(z, w)} F_{0}}\right),
\end{gathered}
$$

i.e., given by substituting $F=F_{0}$ into (28). Substituting $F$ into (21) yields $W^{\lfloor\alpha\rceil}=W_{0}^{\lfloor\alpha\rceil}+W_{1}^{\lfloor\alpha\rceil}+\cdots$ where $W_{1}^{\lfloor\alpha]}=\hat{W}_{1}^{\lfloor\alpha\rceil}\left(F_{1}\right)$ and the map $\hat{W}_{1}: \Gamma \Lambda^{2} M^{+} \rightarrow \Gamma T \mathcal{E}^{+}$is given by

$$
\left.\hat{W}_{1}^{\lfloor\alpha]}\left(F_{1}\right)\right|_{(z, w)}=\frac{q^{\mid \alpha\rceil}}{m^{[\alpha]}} \mathcal{V}_{(z, w)}\left(\widetilde{i_{(z, w)} F_{1}}\right) .
$$

The first order linear system for the perturbation $\left(\theta_{1}, F_{1}\right)$ is then

$$
\begin{aligned}
d \theta_{1}^{\lfloor\alpha\rceil} & =0, \\
i_{W_{0}^{\lfloor\alpha\rceil}} \theta_{1}^{\lfloor\alpha\rceil} & =-i_{\hat{W}_{1}^{\lfloor\alpha]}\left(F_{1}\right)} \theta_{0}^{\lfloor\alpha\rceil}, \\
d F_{1} & =0, \\
\epsilon_{0} d \star F_{1} & =-\sum_{\lfloor\alpha\rceil} q^{\lfloor\alpha\rceil} \oint_{\pi} \theta_{1}^{\lfloor\alpha\rceil} .
\end{aligned}
$$

Using (5) and (38) it follows that each species current in the sum on the right hand side of (41) is closed away from the initial hypersurface $\Sigma_{M}$. In terms of the excitation field $G_{1} \in \Gamma \Lambda^{2} M^{+}$ Eq. (41) will be written

$$
d \star G_{1}=0,
$$

where

$$
G_{1}=\epsilon_{0} F_{1}+\Pi_{1}\left[F_{1}, \zeta_{1}\right]
$$

for some linear functional $\Pi_{1}$ of $F_{1}$ and $\zeta$ such that

$$
d \star \Pi_{1}\left[F_{1}, \zeta_{1}\right]=-\sum_{\lfloor\alpha\rceil} \oint_{\pi} \theta_{1}^{\lfloor\alpha\rceil}
$$

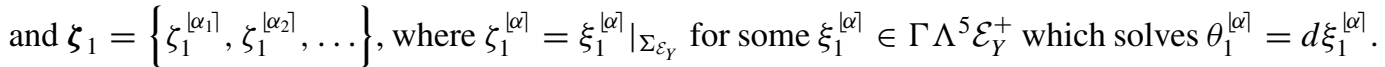
Thus $\zeta_{1}^{[\alpha]}$ is related to the initial velocity profile of the species $\lfloor\alpha\rceil$.

In Sec. III B the general susceptibility kernel $\chi \in \Gamma \Lambda^{0}\left(M_{X}^{+} \times M_{Y}^{+}\right)$and linear functional $Z_{1}$, determined by $\theta_{0}^{\lfloor\alpha\rceil}$ and $F_{0}$, are found such that

$$
\left.\Pi_{1}\left[F_{1}, \zeta_{1}\right]\right|_{x}=\oint_{p_{X}} \chi \wedge p_{Y}^{\star}\left(F_{1}\right)+Z_{1}\left[\zeta_{1}\right]
$$

satisfies (44). 


\section{B. A general formula for the functional $\Pi_{1}$ in an unbounded plasma}

In this section a general expression for a susceptibility kernel will be constructed in terms of the integral curves of the vector field $W_{0}^{[\alpha]} \in \Gamma T \mathcal{E}^{+}$. Such curves describe segments of particle world lines under the influence of the Lorentz force due to the external electromagnetic field $F_{0}$. Although, for a general $F_{0}$, it is not possible to derive an analytic form for such integral curves, special cases are amenable to an analytic analysis.

It proves convenient to let the final and initial states of each species of particle reside in fibers over $M_{X}^{+}$and $M_{Y}^{+}$, respectively, bounded by the equivalent hypersurfaces $\Sigma_{M_{X}} \subset M_{X}^{+}$and $\Sigma_{M_{Y}} \subset M_{Y}^{+}$. Thus the corresponding upper unit hyperboloid bundles $\pi_{X}: \mathcal{E}_{X}^{+} \rightarrow M_{X}^{+}$and $\pi_{Y}: \mathcal{E}_{Y}^{+} \rightarrow M_{Y}^{+}$with boundary hypersurfaces $\Sigma_{\mathcal{E}_{X}} \subset \mathcal{E}_{X}^{+}$and $\Sigma_{\mathcal{E}_{Y}} \subset \mathcal{E}_{Y}^{+}$are used to accommodate the final and initial 4velocities of the particles. The generic elements of these bundles are written $(x, v) \in \mathcal{E}_{X}^{+}$and $(y, u) \in$ $\mathcal{E}_{Y}^{+}$, where $x \in M_{X}^{+}, y \in M_{Y}^{+}$and $g(v, v)=g(u, u)=-1$. The induced coordinate systems for $\mathcal{E}_{X}^{+}$ and $\mathcal{E}_{Y}^{+}$are $\left(x^{0}, \ldots, x^{3}, v^{1}, v^{2}, v^{3}\right)$ and $\left(y^{0}, \ldots, y^{3}, u^{1}, u^{2}, u^{3}\right)$. Let $v^{0}(x, v), v_{0}(x, v), u^{0}(y, u)$, and $u_{0}(y, u)$ be defined in the same way as $w^{0}(z, w)$ and $w_{0}(z, w)$.

The contribution to the tensor $\Pi_{1}\left[F_{1}, \zeta_{1}\right]$ due to all dynamic sources, arises from all particle histories in the past light-cone of $x \in M_{X}^{+}$. The history of the species particle $\lfloor\alpha\rceil$ which passes through event $x$ with 4-velocity $(x, v) \in \mathcal{E}_{X}^{+}$will therefore be parametrized by negative proper time $\tau: C_{(x, v)}^{\lfloor\alpha\rceil}:\left[\tau_{0}^{\lfloor\alpha\rceil}(x, v), 0\right] \rightarrow M^{+}, \tau \mapsto C_{(x, v)}^{\lfloor\alpha\rceil}(\tau)$. Such a history is the unique solution to the Lorentz force equation,

$$
\nabla_{\dot{C}_{(x, v)}^{\lfloor\alpha\rceil}} \dot{C}_{(x, v)}^{\lfloor\alpha\rceil}=\frac{q^{\lfloor\alpha\rceil}}{m^{\lfloor\alpha\rceil}}\left(\widetilde{i_{\dot{C}_{(x, v)}^{\lfloor\alpha\rceil} F_{0}}}\right),
$$

with

$$
g\left(\dot{C}_{(x, v)}^{\lfloor\alpha\rceil}, \dot{C}_{(x, v)}^{\lfloor\alpha\rceil}\right)=-1
$$

and final condition

$$
C_{(x, v)}^{\lfloor\alpha\rceil}(0)=x, \dot{C}_{(x, v)}^{\lfloor\alpha\rceil}(0)=(x, v),
$$

where $\dot{C}_{(x, v)}^{\lfloor\alpha\rceil}(\tau)=C_{(x, v) \star}^{\lfloor\alpha\rceil}\left(\left.\partial_{\tau}\right|_{\tau}\right)=\dot{C}_{(x, v)}^{\lfloor\alpha\rceil a}(\tau) \frac{\partial}{\partial x^{a}}$ and the value $\tau_{0}^{\lfloor\alpha\rceil}(x, v) \leq 0$ solves

$$
C_{(x, v)}^{\lfloor\alpha\rceil}\left(\tau_{0}^{\lfloor\alpha\rceil}(x, v)\right) \in \Sigma_{M_{Y}} .
$$

This defines the prolongation of $C, \dot{C}_{(x, v)}^{\lfloor\alpha\rceil}:\left[\tau_{0}^{\lfloor\alpha\rceil}(x, v), 0\right] \rightarrow \mathcal{E}^{+}$. For each species $\lfloor\alpha\rceil,(x, v) \in$ $\mathcal{E}_{X}^{+}$and $\tau \in\left[\tau_{0}^{\lfloor\alpha\rceil}(x, v), 0\right]$ let $(y, u) \in \mathcal{E}_{Y}^{+}$denote the initial state, i.e., $y=C_{(x, v)}^{\lfloor\alpha\rceil}(\tau)$ and $(y, u)$ $=\dot{C}_{(x, v)}^{\lfloor\alpha\rceil}(\tau)$, see Fig. 1 .

The family of all such histories is described in terms of the maps

$$
\phi^{\lfloor\alpha\rceil}: \mathcal{N}_{X}^{\lfloor\alpha\rceil} \rightarrow \mathcal{E}_{Y}^{+}, \quad \phi^{\lfloor\alpha\rceil}(\tau, x, v)=\dot{C}_{(x, v)}^{\lfloor\alpha\rceil}(\tau),
$$

where

$$
\mathcal{N}_{X}^{\lfloor\alpha\rceil}=\left\{(\tau, x, v) \in \mathbb{R}^{-} \times \mathcal{E}_{X}^{+} \mid \tau_{0}^{\lfloor\alpha\rceil}(x, v) \leq \tau \leq 0\right\}
$$

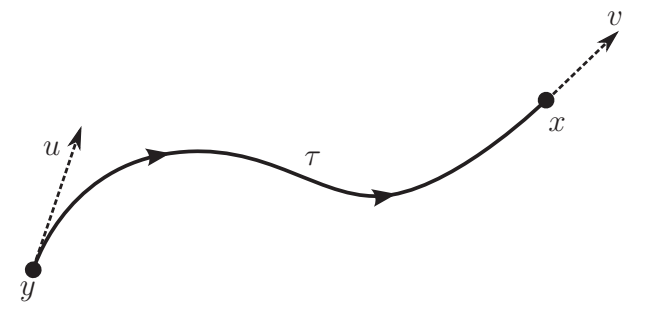

FIG. 1. A segment of the solution curve $C_{(x, v)}$ to the unperturbed Lorentz force equation (46) with final position $x$, final velocity $(x, v)$, initial position $y=C_{(x, v)}(\tau)$, and initial velocity $(y, u)=\dot{C}_{(x, v)}(\tau)$. 
The manifold $\mathcal{N}_{X}^{[\alpha]}$ with boundary is naturally a fiber bundle over $\mathcal{E}_{X}^{+}$with projection $\varpi_{X}^{\lfloor\alpha]}: \mathcal{N}_{X}^{[\alpha]}$ $\rightarrow \mathcal{E}_{X}^{+},(\tau, x, v) \mapsto \varpi_{X}^{\mid \alpha]}(\tau, x, v)=(x, v)$ and for any form $\alpha \in \Gamma \Lambda^{p} \mathcal{N}_{X}$ it follows from (4) that

$$
\oint_{\varpi_{X}^{|\alpha|}} \alpha=d x^{I} \wedge d y^{J} \int_{\tau_{0}^{|\alpha|}(x, v)}^{0} \alpha^{(1)}(\tau, x, v) d \tau
$$

where $\alpha=\alpha^{(1)}(\tau, x, v) d x^{I} \wedge d y^{J} \wedge d \tau+\alpha^{(2)}(\tau, x, v) d x^{I} \wedge d y^{J}$.

Let $\Gamma \Lambda_{\Sigma_{\mathcal{E}_{Y}}}^{5} \mathcal{E}_{Y}^{+}$be the set of sections over $\Sigma_{\mathcal{E}_{Y}}$ with values in $\Lambda^{5} \mathcal{E}_{Y}^{+}$, i.e., if $\alpha \in \Gamma \Lambda_{\Sigma_{\mathcal{E}_{Y}}}^{5} \mathcal{E}_{Y}^{+}$then for each $(y, u) \in \Sigma_{\mathcal{E}_{Y}},\left.\alpha\right|_{(y, u)} \in \Lambda_{(y, u)}^{5} \mathcal{E}_{Y}^{+}$. Let the map $\varphi^{\mid \alpha]}: \Gamma \Lambda_{\Sigma_{\mathcal{E}_{Y}}}^{5} \mathcal{E}_{Y}^{+} \rightarrow \Gamma \Lambda^{5} \mathcal{E}_{X}^{+}$be given by

$$
\left.\varphi^{\lfloor\alpha\rceil}(\alpha)\right|_{(x, v)}=\phi_{\tau_{0}^{[\alpha]}(x, v)}^{\mid \alpha] \star}\left(\left.\alpha\right|_{\tau_{0}^{[\alpha]}(x, v)}\right) \in \Lambda_{(x, v)}^{5} \mathcal{E}_{X}^{+},
$$

where $\phi_{\tau}^{\lfloor\alpha\rceil}: \mathcal{E}_{X}^{+} \rightarrow \mathcal{E}^{+}, \phi_{\tau}^{\lfloor\alpha\rceil}(x, v)=\phi(\tau, x, v)$. For each species $\lfloor\alpha\rceil$ let the initial data be given by $\zeta_{1}^{\lfloor\alpha\rceil} \in \Gamma \Lambda_{\Sigma_{\mathcal{E}_{Y}}}^{5} \mathcal{E}_{Y}^{+}$with $i_{W_{0}^{\lfloor\alpha} \zeta} \zeta_{1}^{\mid \alpha\rceil}=0$.

In terms of these maps, it will now be shown that the general polarization functional $\Pi_{1}$ on $M_{X}^{+}$ is given by

$$
\begin{aligned}
\Pi_{1}\left[F_{1}, \zeta_{1}\right]= & \sum_{\lfloor\alpha\rceil} q^{\lfloor\alpha]} \star \oint_{\pi_{X}} \oint_{\varpi_{X}^{|\alpha|}} d \tau \wedge \phi^{\lfloor\alpha] \star}\left(i_{\hat{W}_{1}^{|\alpha|}\left(F_{1}\right)} \theta_{0}^{\mid \alpha]}\right)+\star d\left(\Xi_{1}\left[F_{1}\right]\right) \\
& +\sum_{\lfloor\alpha\rceil} q^{\lfloor\alpha]} \star \oint_{\pi_{X}} \varphi^{\lfloor\alpha]}\left(\zeta_{1}^{\mid \alpha]}\right)+\star d\left(\check{Z}_{1}\left[\zeta_{1}\right]\right),
\end{aligned}
$$

where $\Xi_{1}$ and $\check{Z}_{1}$ are arbitrary linear functionals of $F_{1}$ and $\zeta_{1}$, respectively. The excitation $\Pi_{1}\left[F_{1}, \zeta_{1}\right]$, in (52), is the general solution to (44) where the source $\theta_{1}$ satisfies (38) and (39). The first two terms on the right hand side of (52) are linear functionals of $F_{1}$ whereas the last term is a linear functional of the initial data $\zeta_{1}$. Clearly $\star d\left(\Xi_{1}\left[F_{1}\right]\right)$ and $\star d\left(\check{Z}_{1}\left[\zeta_{1}\right]\right)$ are in the kernel of $d \star$, the homogeneous differential operator associated with (44).

The proof that (52) solves (44) requires the following lemma which is proved in the Appendix.

Lemma 1: Let $N$ be a manifold with a boundary $\Sigma_{N} \subset N$ and let $V \in \Gamma T N$ be a nonvanishing vector field on $N$ such that every integral curve of $V$ intersects $\Sigma_{N}$ precisely once. For each $\sigma \in N$ let the integral curve of $V$ terminating at $\sigma$ be given by $\gamma_{\sigma}:\left[\tau_{0}(\sigma), 0\right] \rightarrow N$, where $\gamma_{\sigma}(0)=\sigma$ and $\gamma_{\sigma}\left(\tau_{0}(\sigma)\right) \in \Sigma_{N}$. The set $\mathcal{N}=\left\{(\sigma, \tau) \subset \mathbb{R}^{-} \times N \mid \tau_{\min }(\sigma) \leq \tau \leq 0\right\}$ is a fibered manifold over $N$ with projection $\varpi_{N}: \mathcal{N} \rightarrow N,(\tau, \sigma) \mapsto \varpi_{N}(\tau, \sigma)=\sigma$. The family of integral curves of $V$ can be described by the map $\phi_{N}: \mathcal{N} \rightarrow N, \phi_{N}(\tau, \sigma)=\gamma_{\sigma}(\tau)$. Let $\zeta \in \Gamma \Lambda_{\Sigma_{N}}^{p} N$ such that $i_{V} \zeta=0$, i.e., $\zeta$ is a p-form on $\Sigma_{N}$ with values in $\Lambda^{p} N$. Let $\varphi_{N}: \Gamma \Lambda_{\Sigma_{N}}^{p} N \rightarrow \Gamma \Lambda^{p} N$ be given by $\left.\varphi_{N}(\zeta)\right|_{\sigma}=$ $\phi_{N \tau_{0}(\sigma)}^{\star}\left(\left.\zeta\right|_{\tau_{0}(\sigma)}\right) \in \Lambda_{\Sigma_{N}}^{p} N$ the form

If $\beta \in \Gamma \Lambda^{p} N$ is a p-form on $N$ with compact support such that $i_{V} \beta=0$ and $\xi \in \Gamma \Lambda^{p} N$ has

$$
\xi=\oint_{\varpi_{N}} \phi_{N}^{\star}(\beta) \wedge d \tau+\varphi_{N}(\zeta)
$$

then

$$
i_{V} d \xi=\beta
$$

$\left.\xi\right|_{\Sigma_{N}}=\zeta$

This lemma is applied with $N=\mathcal{E}_{X}^{+}, \varpi_{N}=\varpi_{X}^{\lfloor\alpha\rceil}, V=W_{0}^{[\alpha]}, \tau_{0}=\tau_{0}^{\lfloor\alpha\rceil}, \phi_{N}=\phi^{\lfloor\alpha]}, \varphi_{N}=\varphi^{\lfloor\alpha\rceil}$, $\zeta=\zeta_{1}^{\lfloor\alpha\rceil}$, and

$$
\beta=-i_{\hat{W}_{1}^{\lfloor\alpha\rceil}\left(F_{1}\right)} \theta_{0}^{\lfloor\alpha\rceil}
$$

Thus $\xi$ in (53) becomes the 5 -form $\xi_{1}^{\mid \alpha]} \in \Gamma \Lambda^{5} \mathcal{E}_{X}^{+}$,

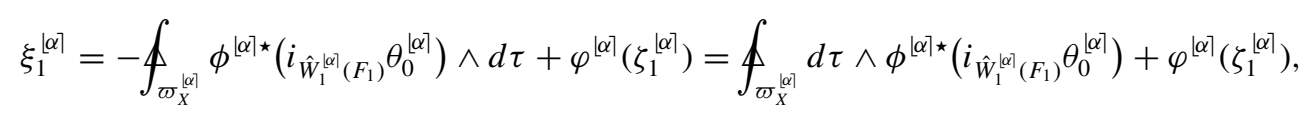


since $\operatorname{deg}\left(\phi^{\lfloor\alpha\rceil \star}\left(i_{\hat{W}_{1}^{\lfloor\alpha]}\left(F_{1}\right)} \theta_{0}^{\lfloor\alpha\rceil}\right)\right)=5$. In order to satisfy (38) let

$$
\theta_{1}^{\lfloor\alpha\rceil}=d \xi_{1}^{\lfloor\alpha\rceil} .
$$

Furthermore, from (54) and (55)

$$
i_{W_{0}^{[\alpha]}} \theta_{1}^{\mid \alpha\rceil}=i_{W_{0}^{[\alpha]}} d \xi_{1}^{\mid \alpha\rceil}=-i_{\hat{W}_{1}^{[\alpha]}\left(F_{1}\right)} \theta_{0}^{\lfloor\alpha\rceil}
$$

so (39) is satisfied. In terms of $\xi_{1}^{\mid \alpha\rceil}$ (52) can be written

$$
\left.\Pi_{1}\left[F_{1}, \zeta_{1}\right]\right|_{x}=\sum_{\lfloor\alpha\rceil} q^{[\alpha]} \star \oint_{\pi_{X}} \xi_{1}^{\mid \alpha\rceil}+\left.\star d\left(\Xi_{1}\left[F_{1}\right]\right)\right|_{x}+\star d\left(\check{Z}_{1}\left[\zeta_{1}\right]\right) .
$$

Then from (5)

$$
\begin{aligned}
d \star \Pi_{1}\left[F_{1}, \zeta_{1}\right] & =d \star \star\left(\sum_{\lfloor\alpha\rceil} q^{\lfloor\alpha\rceil} \oint_{\pi_{X}} \xi_{1}^{\mid \alpha\rceil}\right) \\
& =-\sum_{\lfloor\alpha\rceil} q^{\lfloor\alpha\rceil} d \oint_{\pi_{X}} \xi_{1}^{\lfloor\alpha\rceil}=-\sum_{\lfloor\alpha\rceil} q^{\lfloor\alpha\rceil} \oint_{\pi_{X}} d \xi_{1}^{\mid \alpha\rceil} \\
& =-\sum_{\lfloor\alpha\rceil} q^{\lfloor\alpha\rceil} \oint_{\pi_{X}} \theta_{1}^{\mid \alpha\rceil} .
\end{aligned}
$$

Thus the Maxwell equation (44) is also satisfied. That (52) is the general solution to (44) follows from the fact that the difference between any two solutions of (44) satisfies the homogeneous differential equation associated with (44).

Thus we have succeeded in eliminating $\theta_{1}^{\lfloor\alpha\rceil}$ from the perturbation system (38)-(41), thereby reducing the system to $d F_{1}=0$ and

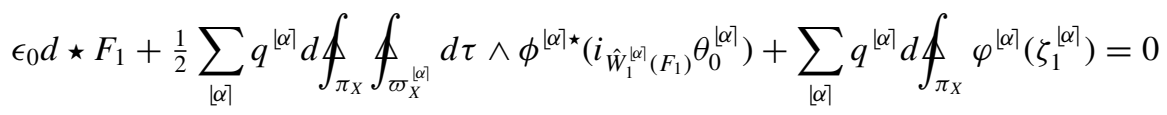

in terms of $\left(\theta_{0}, F_{0}\right)$, for the perturbation $F_{1}$. The perturbation $\theta_{1}$ is then given by (57) and (56).

\section{The susceptibility kernel for an unbounded collisionless plasma}

Equating (52) and (45) with the initial data,

$$
Z_{1}\left[\zeta_{1}\right]=\sum_{\lfloor\alpha\rceil} q^{\lfloor\alpha\rceil} \star \oint_{\pi_{X}} \varphi^{\lfloor\alpha\rceil}\left(\zeta_{1}^{\mid \alpha\rceil}\right)+\star d\left(\check{Z}_{1}\left[\zeta_{1}\right]\right)
$$

yields

$$
\oint_{p_{X}} \chi \wedge p_{Y}^{\star}\left(F_{1}\right)=\sum_{\lfloor\alpha\rceil} q^{\lfloor\alpha]} \star \oint_{\pi_{X}} \oint_{\varpi_{X}^{\mid \alpha\rceil}} d \tau \wedge \phi^{[\alpha] \star}\left(i_{\hat{W}_{1}^{[\alpha]}\left(F_{1}\right)} \theta_{0}^{\lfloor\alpha]}\right)+\star d\left(\Xi_{1}\left[F_{1}\right]\right)
$$

Away from the initial hypersurface boundary $\partial\left(M_{X}^{+} \times M_{Y}^{+}\right)=\Sigma_{M_{X}} \times M_{Y}^{+} \cup M_{X}^{+} \times \Sigma_{M_{Y}}$, using (5) and (A2) one has

$$
\oint_{p_{X}} \star_{X} d_{X} \breve{\xi} \wedge p_{Y}^{\star}\left(F_{1}\right)=\oint_{p_{X}} \star_{X} d \check{\xi} \wedge p_{Y}^{\star}\left(F_{1}\right)=\star d \oint_{p_{X}} \check{\xi} \wedge p_{Y}^{\star}\left(F_{1}\right)=\star d\left(\check{\Xi}_{1}\left[F_{1}\right]\right),
$$

where $\check{\Xi}_{1}\left[F_{1}\right]$ is a linear functional of $F_{1}$. The gauge freedom $\chi \rightarrow \star_{X} d_{X} \breve{\xi}$ given in (10) is equivalent to the addition of the term $\star d\left(\Xi_{1}\left[F_{1}\right]\right)$ in (52).

If $F_{1}$ is restricted to have support in a certain domain one may find $\chi$ such that

$$
\oint_{p_{X}} \chi \wedge p_{Y}^{\star}\left(F_{1}\right)=\sum_{\lfloor\alpha\rceil} q^{\lfloor\alpha]} \star \oint_{\pi_{X}} \oint_{\varpi_{X}^{\mid \alpha]}} d \tau \wedge \phi^{\lfloor\alpha] \star}\left(i_{\hat{W}_{1}^{\lfloor\alpha]}\left(F_{1}\right)} \theta_{0}^{\mid \alpha]}\right)
$$


To find such a susceptibility kernel requires the following maps.

For $(y, u) \in \mathcal{E}_{Y}^{+}$, let $C_{(y, u)}^{\lfloor\alpha\rceil}: \mathbb{R}^{+} \rightarrow M^{+}$and $\dot{C}_{(y, u)}^{\lfloor\alpha]}:\left[0, \tau_{1}^{\lfloor\alpha\rceil}(y, u)\right) \rightarrow \mathcal{E}^{+}$be the unique solutions to the unperturbed Lorentz force equation (46) and (47) with initial conditions

$$
C_{(y, u)}^{\lfloor\alpha\rceil}(0)=y \quad \dot{C}_{(y, u)}^{[\alpha\rceil}(0)=(y, u),
$$

where $\tau_{1}^{[\alpha]}(y, u) \in \mathbb{R}^{+} \cup\{\infty\}$ is the supremum of the values of $\tau$ such that $C_{(y, u)}^{[\alpha]}(\tau) \in M$. Let $\Phi^{\lfloor\alpha]}: \mathcal{N}_{Y}^{[\alpha]} \rightarrow M_{X}^{+} \times M_{Y}^{+}$,

$$
\Phi^{\lfloor\alpha\rceil}(\tau, y, u)=\left(C_{(y, u)}^{\lfloor\alpha\rceil}(\tau), y\right)
$$

where

$$
\mathcal{N}_{Y}^{\lfloor\alpha\rceil}=\left\{(\tau, y, u) \in \mathbb{R}^{+} \times \mathcal{E}_{X}^{+} \mid 0 \leq \tau<\tau_{1}^{\mid \alpha\rceil}(y, u)\right\} .
$$

This map gives the final and initial positions of a solution to the unperturbed Lorentz force equation in terms of the initial position, velocity, and proper time parameter $\tau \in\left[0, \tau_{1}^{|\alpha\rangle}(y, u)\right)$.

Observe that $\Phi^{\lfloor\alpha]}$ is never surjective, since if $\Phi^{[\alpha]}(\tau, y, u)=(x, y)$ then $x \in J^{+}(y)$. Also $\Phi^{\lfloor\alpha]}$ is never injective since $\Phi^{[\alpha]}(0, y, u)=(y, y)$ for all $(y, u) \in \mathcal{E}_{Y}^{+}$. Thus $\Phi^{[\alpha]}$ does not possess an inverse and one must work locally on $M_{X}^{+} \times M_{Y}^{+}$in order to establish the diffeomorphism $\Psi^{\lfloor\alpha\rceil}: \mathcal{D} \rightarrow \mathcal{D}^{\prime}$,

$$
\Psi^{\mid \alpha\rceil}=\left(\Phi^{\mid \alpha\rceil} \mid \mathcal{D}^{\prime}\right)^{-1},
$$

i.e.,

$$
\Psi^{\lfloor\alpha\rceil}\left(C_{(y, u)}(\tau), y\right)=(\tau, y, u)
$$

with $\mathcal{D} \subset M_{X}^{+} \times M_{Y}^{+}$and $\mathcal{D}^{\prime} \subset \mathcal{N}_{Y}^{\lfloor\alpha\rceil}$ given by

$$
\mathcal{D}=\left\{(x, y) \mid \text { There exists a unique } u \in \mathcal{E}_{y} \text { and } \tau \in \mathbb{R}^{+} \text {such that } C_{(y, u)}^{\lfloor\alpha]}(\tau)=x \text { for all }\lfloor\alpha\rceil\right\},
$$

$$
\mathcal{D}^{\prime}=\left\{(\tau, y, u) \mid \Phi^{\lfloor\alpha\rceil}(\tau, y, u) \in \mathcal{D} \text { for all }\lfloor\alpha\rceil\right\} .
$$

This map $\Psi^{\lfloor\alpha]}$ encodes the solution to the 2-point problem, namely given an initial event $y \in M_{Y}$ and final event $x \in M_{X}$ find the unique worldline to the unperturbed Lorentz force equation which passes though these two points. This worldline is specified by its initial velocity $(y, u) \in \mathcal{E}_{X}^{+}$and its proper time $\tau$. The statement that $\Phi^{[\alpha]}$ does not have an inverse is equivalent to the statement that in general there may not be a unique solution to the two point problem on an arbitrary domain. The domain $\mathcal{D}$ is the set of all pairs $(x, y)$ such that there is a unique worldline.

Set

$$
\chi=\sum_{\lfloor\alpha\rceil} \chi^{\lfloor\alpha\rceil}
$$

where

$$
\left.\chi^{\lfloor\alpha]}\right|_{(x, y)}=\left.\frac{1}{2} \frac{q^{\lfloor\alpha\rceil 2}}{m^{[\alpha\rceil}} \star_{X} d y^{c d} \wedge i_{a b c d}^{(y)} \Psi^{\lfloor\alpha\rceil \star}\left(d \tau \wedge \varpi_{Y}^{\lfloor\alpha\rceil \star}\left(g^{v a} u^{b} i_{v}^{(u)} \theta_{0}^{\lfloor\alpha\rceil}\right)\right)\right|_{(x, y)}
$$

for points $(x, y) \in \mathcal{D}$. In the Appendix (Lemma 6) it is shown that given $x \in M_{X}^{+}(61)$ and $F_{1}$ with support in

$$
\mathcal{D}_{x}=\mathcal{D} \cap p_{X}^{-1}\{x\}=\left\{y \in M_{Y} \mid(x, y) \in \mathcal{D}\right\}
$$

then (61) holds at $x$. Furthermore, although $\left.\left(d_{Y} \chi\right)\right|_{(x, y)}$ is unique, $\chi$ has the gauge freedom given by (9).

One may write (67) implicitly as

$$
\chi^{\lfloor\alpha\rceil} \wedge p_{Y}^{\star} \gamma=-q^{[\alpha]} \star_{X} S \Psi^{\lfloor\alpha\rceil \star}\left(d \tau \wedge \varpi_{Y}^{\lfloor\alpha\rceil \star}\left(i_{\hat{W}_{1}[\alpha]}(\gamma) \theta_{0}^{\lfloor\alpha\rceil}\right)\right)
$$


for all $\gamma \in \Gamma \Lambda^{2} M_{Y}^{+}$where $S: \Lambda_{(x, y)}^{6}\left(M_{X}^{+} \times M_{Y}^{+}\right) \rightarrow \Lambda_{(x, y)}^{6}\left(M_{X}^{+} \times M_{Y}^{+}\right)$,

$$
S(\alpha)=i_{0123}^{(y)} \alpha \wedge d y^{0123} .
$$

The tensor projector $S$ has the simplest representation in the coordinate basis employed here since $i_{a}^{(y)} d y^{b}=\delta_{a}^{b}$.

From (64) for a chosen species $\lfloor\alpha\rceil$ one must consider $\tau$ and $u$ to be functions of $(x, y)$ as well as the species label $\lfloor\alpha\rceil$. Thus let $\Psi\lfloor\alpha\rceil$ be given by the functions $\tau=\tau(x, y)$ and $u^{\mu}=u^{\mu}(x, y)$, where we have dropped the species label, i.e., $\tau(x, y)$ and $u^{\mu}(x, y)$ solve the implicit equation

$$
C_{(y, u(x, y))}^{\lfloor\alpha\rceil}(\tau(x, y))=x
$$

where $u^{0}(x, y)$ is the solution to $u^{a}(x, y) u^{b}(x, y) g_{a b}(y)=-1$ and $u_{0}(x, y)=g_{a 0}(y) u^{a}(x, y)$. Let $f_{0}^{[\alpha]}=f_{0}^{\lfloor\alpha]}(y, u)$ represent the unperturbed probability function on $\mathcal{E}_{Y}^{+}$. The contribution to the susceptibility kernel from species $\lfloor\alpha\rceil$ is given in local coordinates by (Lemma 7 in the Appendix.)

$$
\begin{aligned}
&\left.\chi^{[\alpha]}\right|_{(x, y)}=-f_{0}^{\lfloor\alpha\rceil} \frac{q^{[\alpha\rceil 2}}{m^{[\alpha]}} \frac{|\operatorname{det} g|^{3 / 2}}{4 u_{0}} g^{\mu c} u^{b} \epsilon^{d e j k} \epsilon_{c b i h} \epsilon_{\mu \nu \sigma} \times \\
&\left(\frac{u^{a}}{2} \frac{\partial \tau}{\partial y^{a}} \frac{\partial u^{v}}{\partial x^{d}} \frac{\partial u^{\sigma}}{\partial x^{e}}-\frac{u^{a}}{2} \frac{\partial \tau}{\partial x^{d}} \frac{\partial u^{v}}{\partial y^{a}} \frac{\partial u^{\sigma}}{\partial x^{e}}+\frac{u^{a}}{2} \frac{\partial \tau}{\partial x^{d}} \frac{\partial u^{v}}{\partial x^{e}} \frac{\partial u^{\sigma}}{\partial y^{a}}\right. \\
&\left.+\left(-\Gamma^{v}{ }_{p f} u^{p} u^{f}+\frac{q^{\lfloor\alpha\rceil}}{m^{[\alpha]}} F_{0 p f} g^{v p} u^{f}\right) \frac{\partial \tau}{\partial x^{d}} \frac{\partial u^{\sigma}}{\partial x^{e}}\right) d x_{j k} \wedge d y^{i h},
\end{aligned}
$$

where $g, F_{0}$, and $\Gamma^{v}$ ef are all evaluated at $y \in M_{Y}^{+}$and each $\tau$ and $u$ belongs to the species $\lfloor\alpha\rceil$. This is a key result of our article.

\section{A spacetime inhomogeneous microscopically neutral plasma}

In a Vlasov model, a plasma or gas is deemed microscopically neutral if in its unperturbed state $F_{0}=0$. Let $M$ be Minkowski spacetime with global Lorentzian coordinates so that $\Gamma_{a b}^{v}=0$. Assume that $f_{0}^{\lfloor\alpha\rceil}$ solves the zeroth order Maxwell-Vlasov system (35) with $\theta_{0}^{\lfloor\alpha\rceil}=i_{W_{0}^{\mid \alpha\rceil}}\left(f_{0}^{\lfloor\alpha\rceil} \Omega\right.$ ) and $F_{0}=0$. In this scenario one can calculate $\chi$ explicitly.

Since Minkowski spacetime is flat and $F_{0}=0$ the integral curves $C_{(x, v)}$ in global Lorentzian coordinates are the straight lines

$$
\tau=\sqrt{-g(x-y, x-y)} \quad u=\frac{(x-y)}{\tau} .
$$

Differentiating with respect to $x^{a}$ and $y^{a}$ gives

$$
\frac{\partial \tau}{\partial x^{a}}=-u_{a}, \quad \frac{\partial \tau}{\partial y^{a}}=u_{a}, \quad \frac{\partial u^{a}}{\partial x^{b}}=\frac{\left(\delta_{b}^{a}+u_{a} u_{b}\right)}{\tau}, \quad \frac{\partial u^{a}}{\partial y^{b}}=-\frac{\left(\delta_{b}^{a}+u_{a} u_{b}\right)}{\tau} .
$$

If follows from (72) that

$$
\left.\chi^{[\alpha]}\right|_{(x, y)}=\frac{q^{\lfloor\alpha]} f_{0}^{\mid \alpha\rceil}(y, u)}{4 u_{0} \tau^{2}} g^{\mu c} u^{b} \epsilon_{c b i h}\left(2 d x_{0 \mu}+\epsilon^{d \sigma j k} \epsilon_{\mu v \sigma} u^{v} u_{d} d x_{j k}\right) \wedge d y^{i h},
$$

where $\tau(x, y)$ and $u(x, y)$ are given by (73).

It is often useful to explore the response of an inhomogeneous plasma due to a monochromatic electromagnetic plane wave with constant amplitude $E$,

$$
F_{1}=E e^{-i \omega x^{0}+i k x^{1}} d x^{01} .
$$

Setting the initial hypersurface as $\Sigma_{\mathcal{E}_{Y}}=\left\{y^{0}=y_{0}^{0}\right\}$, the general initial 5-form $\zeta_{1}^{[\alpha]} \in \Gamma \Lambda_{\Sigma_{\mathcal{E}_{Y}}}^{5} \mathcal{E}_{Y}^{+}$ satisfying $i_{W_{0}} \zeta_{1}^{\lfloor\alpha]}=0$ is given in terms of its components by

$$
\begin{gathered}
\left.\zeta_{1}^{\lfloor\alpha\rceil}\right|_{\left(0, y^{\mu}, u^{v}\right)}=\left(u^{0} d y^{1}-u^{1} d y^{0}\right) \wedge\left(\zeta_{1,1}^{\lfloor\alpha\rceil} d y^{2} \wedge d u^{123}+\zeta_{1,2}^{\lfloor\alpha]} d y^{3} \wedge d u^{123}\right)+\zeta_{1,3}^{\lfloor\alpha]} d y^{23} \wedge d u^{123} \\
+\left(u^{0} d y^{123}-u^{1} d y^{023}\right)\left(\zeta_{1,4}^{\mid \alpha\rceil} d u^{12}+\zeta_{1,5}^{\lfloor\alpha]} d u^{13}+\zeta_{1,6}^{[\alpha]} d u^{23}\right),
\end{gathered}
$$


where $\zeta_{1, A}^{\lfloor\alpha]}=\check{\zeta}_{1, A}^{\lfloor\alpha\rceil}\left(y^{\mu}, u^{v}\right)$ for $A=1, \ldots 6$. For the integral curves (73) and the initial hypersurface $\Sigma_{\mathcal{E}_{Y}}=\left\{y^{0}=y_{0}^{0}\right\}$ one has $\tau_{0}(x, v)=\left(y_{0}^{0}-x^{0}\right) / v^{0}$ and the map $\varphi$ is given by (51) with $\phi_{\tau}^{\star}\left(y^{a}\right)=$ $x^{a}+\tau y^{a}$ and $\phi_{\tau}^{\star}\left(u^{a}\right)=v^{a}$. From (45) with $\chi$ given by (75) and $Z_{1}\left[\zeta_{1}\right]$ given by (59) one has

$$
\begin{aligned}
& \Pi_{1}\left[F_{1}, \zeta_{1}\right]= \\
& -\sum_{\lfloor\alpha\rceil} \frac{q^{\mid \alpha\rceil 2}}{m^{\lfloor\alpha\rceil}} E e^{-i \omega x^{0}+i k x^{1}}\left\{d x^{01} \int d v^{123} T^{\lfloor\alpha\rceil} \frac{\left(v^{0}\right)^{2}-\left(v^{1}\right)^{2}}{v^{0}}+d x^{12} \int d v^{123} T^{\lfloor\alpha\rceil} v^{2}\right. \\
& \left.-d x^{02} \int d v^{123} T^{\lfloor\alpha\rceil} \frac{v^{2} v^{1}}{v^{0}}+d x^{13} \int d v^{123} T^{[\alpha\rceil} v^{3}+d x^{03} \int d v^{123} T^{\lfloor\alpha\rceil} \frac{v^{3} v^{1}}{v^{0}}\right\} \\
& +\sum_{\lfloor\alpha\rceil} q^{[\alpha\rceil}\left\{d x^{02} \int d v^{123}\left(\zeta_{1,4}^{\lfloor\alpha]} \frac{v^{1}\left(x^{0}-y_{0}^{0}\right)}{v^{0}}-\zeta_{1,1}^{\mid \alpha\rceil} v^{1}\right)\right. \\
& +d x^{03} \int d v^{123}\left(\zeta_{1,5}^{\lfloor\alpha]} \frac{v^{1}\left(x^{0}-y_{0}^{0}\right)}{v^{0}}-\zeta_{1,2}^{\lfloor\alpha\rceil} v^{1}\right) \\
& +d x^{12} \int d v^{123}\left(v^{0} \zeta_{1,1}^{\lfloor\alpha]}-\zeta_{1,4}^{\lfloor\alpha]}\left(x^{0}-y_{0}^{0}\right)\right)+d x^{13} \int d v^{123}\left(v^{0} \zeta_{1,2}^{\lfloor\alpha]}-\zeta_{1,5}^{\lfloor\alpha]}\left(x^{0}-y_{0}^{0}\right)\right) \\
& \left.+d x^{23} \int d v^{123}\left(\zeta_{1,3}^{\mid \alpha\rceil}+\zeta_{1,4}^{\mid \alpha\rceil} \frac{v^{1} v^{3}\left(x^{0}-y_{0}^{0}\right)}{\left(v^{0}\right)^{2}}-\zeta_{1,5}^{\mid \alpha\rceil} \frac{v^{1} v^{2}\left(x^{0}-y_{0}^{0}\right)}{\left(v^{0}\right)^{2}}+\zeta_{1,6}^{\lfloor\alpha\rceil}\left(x^{0}-y_{0}^{0}\right)\left(\frac{v^{1}}{v^{0}}-1\right)\right)\right\} \\
& +\star d\left(\Xi_{1}\left[F_{1}\right]\right)+\star d\left(\check{Z}_{1}\left[\zeta_{1}\right]\right) \text {, }
\end{aligned}
$$

where $\int d v^{123}$ denotes the triple integral operator $\iiint_{-\infty}^{\infty} d v^{123}, v^{0}=\sqrt{1+v_{\mu} v^{\mu}}$,

$$
T^{[\alpha]}=T^{\lfloor\alpha\rceil}(x, v)=\int_{\left(y_{0}^{0}-x^{0}\right) / v^{0}}^{0} e^{i \tau\left(-\omega v^{0}+k v^{1}\right)} f_{0}^{\lfloor\alpha]}(x+\tau v, v) \tau d \tau,
$$

and $\zeta_{1, A}^{[\alpha]}=\zeta_{1, A}^{[\alpha]}\left(x^{\mu}, v^{\mu}\right)=\check{\zeta}_{1, A}^{\lfloor\alpha]}\left(x^{\mu}-x^{0} v^{\mu} / v^{0}, v^{v}\right)$ in (78). This response is not in general plane fronted.

For the particular case of a plane fronted plasma distribution,

$$
f_{0}^{\lfloor\alpha\rceil}(x, v)=h_{0}^{\lfloor\alpha\rceil}\left(x^{0}, x^{1}, v^{1}\right) \delta\left(v^{2}\right) \delta\left(v^{3}\right),
$$

with initial data,

$$
\zeta_{1}^{\lfloor\alpha\rceil}=0
$$

(78) becomes the plane fronted 2-form,

$$
\begin{aligned}
&\left.\Pi_{1}\left[F_{1}, \zeta\right]\right|_{x} \\
&=-d x^{01} \sum_{\lfloor\alpha\rceil} \frac{q^{\lfloor\alpha\rceil 2}}{m^{\lfloor\alpha]}} E e^{-i \omega x^{0}+i k x^{1}} \int_{-\infty}^{\infty} d v^{1} \int_{\left(y_{0}^{0}-x^{0}\right) / v^{0}}^{0} d \tau e^{i \tau\left(-\omega v^{0}+k v^{1}\right)} h_{0}^{\lfloor\alpha\rceil}\left(x^{0}+\tau v^{0}, x^{1}+\tau v^{1}, v^{1}\right) \frac{\tau}{v^{0}} \\
&+\star d\left(\Xi_{1}\left[F_{1}\right]\right)
\end{aligned}
$$

describing the response of a spacetime inhomogeneous unbounded plasma to (76).

\section{E. Spacetime homogeneous unbounded plasmas}

The previous discussion simplifies considerably if the unperturbed plasmas is homogeneous in space and time. In Minkowski spacetime $M$, an unbounded unperturbed plasma is deemed spacetime homogeneous if $A_{z}^{\star} F_{0}=F_{0}$ and $\dot{A}_{z}^{\star} \theta_{0}^{\lfloor\alpha\rceil}=\theta_{0}^{\lfloor\alpha\rceil}$ for all $z \in M$, where the translation map $A_{z}: M \rightarrow M$, $A_{z}(x)=x+z$ induces the map $\dot{A}_{z}: \mathcal{E} \rightarrow \mathcal{E}, \dot{A}_{z}=A_{z \star}$. Such spacetime homogeneity implies that 
in all inertial frames the medium is stationary and spatially homogeneous in all directions. Such a spacetime homogeneous plasma will give rise to a spacetime homogeneous electromagnetic constitutive relation. In addition to the components $\left(F_{0}\right)_{a b}$ with respect to an inertial frame being constant, the functions $f^{\lfloor\alpha\rceil}(x, v)$ are independent of event position $x$ and can therefore be written $f^{\lfloor\alpha\rceil}(v)$.

In this scenario the Fourier transform (17) of the susceptibility kernel (18) for each species, is then given by

$$
\begin{aligned}
& \hat{\chi}^{\lfloor\alpha\rceil}{ }_{a b}{ }^{e f}(k) d x^{a b} \\
& \quad=\frac{1}{2} q^{\lfloor\alpha\rceil} d x_{g h} \int_{-\infty}^{0} d \tau \int d v^{123} f_{0}^{\lfloor\alpha\rceil}(v) e^{-i k \cdot \boldsymbol{L}^{\lfloor\alpha\rceil} v} \frac{v^{g}}{v_{0}}\left(g^{\nu e} u^{f}-g^{\nu f} u^{e}\right)\left(\boldsymbol{L}^{\lfloor\alpha\rceil}{ }_{\nu}{ }^{2}(\tau)-\frac{u_{v}}{u_{0}} \boldsymbol{L}_{0}^{\lfloor\alpha\rceil}{ }^{h}(\tau)\right),
\end{aligned}
$$

where $\boldsymbol{F}_{0}$ is the $4 \times 4$ real matrix with components $\left(\boldsymbol{F}_{0}\right)^{a}{ }_{b}=\eta^{a c}\left(F_{0}\right)_{c b}$ generating the matrices

$$
\begin{array}{ll}
\boldsymbol{D}^{\lfloor\alpha\rceil^{a}}{ }_{b}(\tau)=\exp \left(\tau \frac{q^{\lfloor\alpha\rceil}}{{ }^{\lfloor\alpha\rceil}} \boldsymbol{F}_{0}\right)_{b}^{a}, & \boldsymbol{D}_{b}^{\lfloor\alpha\rceil}{ }_{b}^{a}(\tau)=g_{b c} \boldsymbol{D}^{\left\lfloor\alpha{ }^{c}\right.}{ }_{d}(\tau) g^{d a}, \\
\boldsymbol{L}^{\left\lfloor\alpha{ }^{a}\right.}{ }_{b}(\tau)=\int_{0}^{\tau} \boldsymbol{D}^{\lfloor\alpha\rceil^{a}}{ }_{b}\left(\tau^{\prime}\right) d \tau^{\prime}, & \boldsymbol{L}_{b}^{\lfloor\alpha\rceil}{ }_{b}^{a}(\tau)=g_{b c} \boldsymbol{L}^{\lfloor\alpha\rceil^{c}}{ }_{d}(\tau) g^{d a},
\end{array}
$$

$k \cdot \boldsymbol{L}^{\lfloor\alpha\rceil} v=k_{a} \boldsymbol{L}^{\lfloor\alpha\rceil}{ }_{b}(\tau) v^{b}$,

$$
u^{a}\left(\tau, v^{1}, v^{2}, v^{3}\right)=\boldsymbol{D}^{\lfloor\alpha\rceil^{a}}{ }_{b}(\tau) v^{b} .
$$

The susceptibility kernel (82) can be shown to agree with the results of O'Sullivan and Derfler. ${ }^{12}$

Furthermore, for a microscopically neutral spacetime homogeneous plasma with $F_{0}=0$, $G_{1}=0$, and $f_{0}^{\lfloor\alpha\rceil}(v)=h_{0}^{\lfloor\alpha\rceil}\left(v^{1}\right) \delta\left(v^{2}\right) \delta\left(v^{3}\right)$ it follows from (81) and (43) that for $\operatorname{Im}(\omega)>0$,

$$
1=\sum_{\lfloor\alpha\rceil} \frac{q^{\lfloor\alpha\rceil 2}}{m^{\lfloor\alpha\rceil} \epsilon_{0}} \int_{-\infty}^{\infty} \frac{h_{0}^{\lfloor\alpha\rceil}\left(v^{1}\right) d v^{1}}{v^{0}\left(-\omega v^{0}+k v^{1}\right)^{2}} .
$$

The relativistic Landau damped dispersion relation for plane fronted Langmuir modes in an unperturbed spacetime homogeneous plasma arises by analytic continuation of the integral (85) to the lower-half complex $\omega$ plane.

\section{F. Langmuir modes for an inhomogeneous unbounded plasma in Minkowski spacetime}

If the plasma is microscopically neutral but spacetime inhomogeneous in its unperturbed state the Landau dispersion relation corresponding to (85) becomes more involved. We define the generalized Langmuir sector to contain perturbations described by (81) but with the external polarization specified by $\Xi_{1}\left[F_{1}\right]$ set to zero. Since $\zeta_{1}^{[\alpha]}=0, \Pi_{1}\left[F_{1}, 0\right]$ will be denoted $\Pi_{1}\left[F_{1}\right]$. Thus (43) with $G_{1}=0$ becomes

$$
\epsilon_{0} F_{1}=-\Pi_{1}\left[F_{1}\right]
$$

Consider the case where planar inhomogeneities in a plasma composed of electrons and ions arise from the unperturbed spacetime inhomogeneous solution to the Maxwell-Vlasov system Eqs. (35) and (36) with $F_{0}=0$ and

$$
\begin{aligned}
f_{0}^{\text {|e巾 }}\left(x^{0}, x^{1}, x^{2}, x^{3}, v^{1}, v^{2}, v^{3}\right) & =f_{0}^{\text {[ion }\rceil}\left(x^{0}, x^{1}, x^{2}, x^{3}, v^{1}, v^{2}, v^{3}\right) \\
& =h\left(x^{1}-\frac{v^{1} x^{0}}{v^{0}}, v^{1}\right) \delta\left(v^{2}\right) \delta\left(v^{3}\right),
\end{aligned}
$$

where $q^{\lfloor\mathrm{e}\rceil}=-q^{\text {[ion] }}$. 
For example, one might consider

$$
h\left(x^{1}, v^{1}\right)=n^{\text {[ion }]}\left(x^{1}\right) A^{[\text {ion }]}\left(x^{1}\right) \exp \left(-\frac{m^{\text {[ion }]} v^{0}}{k_{B} T^{\text {[ion }]}\left(x^{1}\right)}\right),
$$

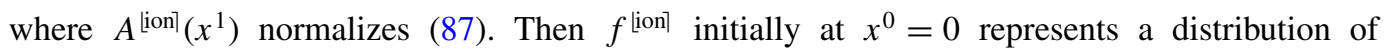
ions where, at each spatial point $x^{1}$, the velocities belong to the one-dimensional MaxwellJüttner distribution. In such a distribution the temperature $T^{[\text {ion }]}\left(x^{1}\right)$ and the number den-

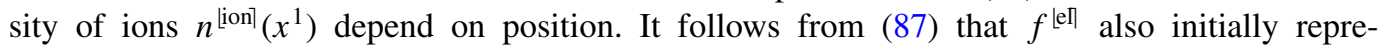
sents a position dependent Maxwell-Jüttner distribution, where $n^{\lfloor\mathrm{e}\rceil}\left(x^{1}\right)=n^{[\mathrm{ion}\rceil}\left(x^{1}\right)$ and $T^{\text {[eा }}\left(x^{1}\right)$ $=T^{\text {[ion] }}\left(x^{1}\right) m^{\text {[eा] }} / m^{\text {[ion] }}$. After the initial moment, the ions and electrons drift according to (87) and velocities do not remain in the Maxwell-Jüttner distributions. Alternatively (87) might describe a plasma composed of particles and antiparticles.

In the theory of a spacetime homogeneous plasma $\omega$ and $k$ satisfy the transcendental dispersion relation (85). This relation contains an integral that is potentially singular. The Landau prescription circumvents this singularity by complexifying $\omega$ and defining an analytic continuation for the integral in the complex $\omega$ plane.

Setting $h_{0}^{\lfloor\alpha\rceil}\left(x^{0}, x^{1}, v^{1}\right)=h\left(x^{1}-v^{1} x^{0} / v^{0}, v^{1}\right)$ in (80) yields (87) and (81) becomes

$$
\begin{aligned}
& \left.\Pi_{1}\left[F_{1}\right]\right|_{x} \\
& =-d x^{01} q^{\lfloor\mathrm{el} \mid 2}\left(\frac{1}{m^{\text {[ion] }}}+\frac{1}{m^{\text {[e巾 }}}\right) E e^{-i \omega x^{0}+i k x^{1}} \int_{-\infty}^{\infty} d v^{1} h\left(x^{1}-\frac{v^{1} x^{0}}{v^{0}}, v^{1}\right) \int_{\left(y_{0}^{0}-x^{0}\right) / v^{0}}^{0} d \tau e^{i \tau\left(-\omega v^{0}+k v^{1}\right)} \frac{\tau}{v^{0}} .
\end{aligned}
$$

To compare with the results (85) given for the homogeneous case, consider the limit $y_{0}^{0} \rightarrow-\infty$ with $\operatorname{Im}(\omega)>0$. Furthermore, for the nonevanescent modes considered here $\operatorname{Im}(k)=0$. Thus (88) becomes

$$
\left.\Pi_{1}\left[F_{1}\right]\right|_{x}=-d x^{01} \epsilon_{0} \mathcal{Q}_{0}^{2} E e^{-i \omega x^{0}+i k x^{1}} \int_{-\infty}^{\infty} d v^{1} \frac{h\left(x^{1}-v^{1} x^{0} / v^{0}, v^{1}\right)}{v^{0}\left(-\omega v^{0}+k v^{1}\right)^{2}}
$$

where

$$
\mathcal{Q}_{0}^{2}=\frac{q^{\mathrm{Le}\rceil 2}}{\epsilon_{0} m^{\lfloor\text {ion }\rceil}}+\frac{q^{\mathrm{ee}\rceil 2}}{\epsilon_{0} m^{\lfloor\mathrm{e}\rceil}} .
$$

In a spacetime inhomogeneous plasma there is no time-harmonic solution or associated transcendental dispersion relation between $\omega$ and $k$. We therefore propose solving (86) with a longitudinal field $F_{1}$ represented as the packet

$$
F_{1}\left(x^{0}, x^{1}\right)=d x^{01} \int_{-\infty}^{\infty} d \hat{\omega} \int_{-\infty}^{\infty} d \hat{k} \hat{E}(\hat{\omega}, \hat{k}) e^{-i \hat{\omega} x^{0}+i \hat{k} x^{1}}
$$

Substituting (89) and (90) into (86) yields

$$
\begin{aligned}
& \int_{-\infty}^{\infty} d \hat{\omega} \int_{-\infty}^{\infty} d \hat{k} \hat{E}(\hat{\omega}, \hat{k}) e^{-i \hat{\omega} x^{0}+i \hat{k} x^{1}} \\
& \quad=\mathcal{Q}_{0}^{2} \int_{-\infty}^{\infty} d \hat{\omega} \int_{-\infty}^{\infty} d \hat{k} \hat{E}(\hat{\omega}, \hat{k}) e^{-i \hat{\omega} x^{0}+i \hat{k} x^{1}} \int_{-\infty}^{\infty} d v^{1} \frac{h\left(x^{1}-v^{1} x^{0} / v^{0}, v^{1}\right)}{v^{0}\left(\hat{\omega} v^{0}+\hat{k} v^{1}\right)^{2}}
\end{aligned}
$$

Performing the inverse Fourier transform gives

$$
\begin{aligned}
& 4 \pi^{2} \hat{E}(\omega, k) \\
& =\mathcal{Q}_{0}^{2} \int_{-\infty}^{\infty} d x^{0} \int_{-\infty}^{\infty} d x^{1} \int_{-\infty}^{\infty} d \hat{\omega} \int_{-\infty}^{\infty} d \hat{k} \hat{E}(\hat{\omega}, \hat{k}) e^{i\left(-(\hat{\omega}-\omega) x^{0}+(\hat{k}-k) x^{1}\right)} \int_{-\infty}^{\infty} d v^{1} \frac{h\left(x^{1}-v^{1} x^{0} / v^{0}, v^{1}\right)}{v^{0}\left(\hat{\omega} v^{0}+\hat{k} v^{1}\right)^{2}} .
\end{aligned}
$$


Since

$$
\begin{aligned}
& \int_{-\infty}^{\infty} d x^{0} \int_{-\infty}^{\infty} d x^{1} e^{i\left(-(\hat{\omega}-\omega) x^{0}+(\hat{k}-k) x^{1}\right)} h\left(x^{1}-v^{1} x^{0} / v^{0}, v^{1}\right) \\
& =2 \pi \hat{h}\left(k-\hat{k}, v^{1}\right) \delta\left(\hat{\omega}-\omega+v^{1}(k-\hat{k}) / v^{0}\right)
\end{aligned}
$$

where

$$
\hat{h}\left(k, v^{1}\right)=\int_{-\infty}^{\infty} e^{-i k s} h\left(s, v^{1}\right) d s
$$

one has

$$
\hat{E}(\omega, k)=\frac{\mathcal{Q}_{0}^{2}}{2 \pi} \int_{-\infty}^{\infty} d \hat{\omega} \int_{-\infty}^{\infty} d \hat{k} \int_{-\infty}^{\infty} d v^{1} \frac{\hat{E}(\hat{\omega}, \hat{k})}{v^{0}\left(\hat{\omega} v^{0}+\hat{k} v^{1}\right)^{2}} \hat{h}\left(k-\hat{k}, v^{1}\right) \delta\left(\hat{\omega}-\omega+v^{1}(k-\hat{k}) / v^{0}\right) .
$$

Since we restrict to nonevanescent modes $k$ and $\hat{k}$ are real. For $\hat{E}(\omega, k)$ to be nonzero one requires the argument of the $\delta$-function to be zero. Since $v^{1}$ is real and therefore $v^{1}(k-\hat{k}) / v^{0}$ is real it follows that although $\operatorname{Im}(\omega)>0$ and $\operatorname{Im}(\hat{\omega})>0$ the difference $\omega-\hat{\omega}$ is real. Furthermore, from $\hat{\omega}-\omega+v^{1}(k-\hat{k}) / v^{0}=0$ it follows that $|\hat{\omega}-\omega|<|\hat{k}-k|$. Thus (91) becomes

$$
\hat{E}(\omega, k)=\frac{\mathcal{Q}_{0}^{2}}{2 \pi} \int_{-\infty}^{\infty} d \hat{k} I(\omega, k, \hat{k})
$$

where

$$
I(\omega, k, \hat{k})=\int_{S(\omega, k, \hat{k})} d \hat{\omega} \hat{E}(\hat{\omega}, \hat{k}) \frac{(k-\hat{k})}{(\hat{\omega} k-\hat{k} \omega)^{2}} \hat{h}\left(k-\hat{k}, \frac{k-\hat{k}}{\sqrt{(\hat{k}-k)^{2}-(\hat{\omega}-\omega)^{2}}}\right)
$$

and the contour of integration for $\hat{\omega}$ in (93) is the straight line $S(\omega, k, \hat{k})$ where $\operatorname{Im}(\hat{\omega})=\operatorname{Im}(\omega)>0$ and $-|\hat{k}-k|<\operatorname{Re}(\hat{\omega}-\omega)<|\hat{k}-k|$. Since $(\hat{\omega}-\omega)^{2}<(\hat{k}-k)^{2}$ the arguments of $\hat{h}$ in (93) are always real and nonsingular on $S(\omega, k, \hat{k})$.

To accommodate the situation when $\hat{E}(\omega, k)$ describes damped electromagnetic waves one must continue (93) to $\operatorname{Im}(\omega)<0$ for real $k$. However, there is a double pole in the complex $\hat{\omega}$ plane at $\hat{\omega}=\hat{\omega}_{0}=\hat{k} \omega / k$ that coincides with $S(\omega, k, \hat{k})$ when $\operatorname{Im}(\omega)=0$ and $|\omega|<|k|$. To define an analytic continuation of (93) to $\operatorname{Im}(\omega)<0$ when $|\operatorname{Re}(\omega)|<|k|$, we indent $S(\omega, k, \hat{k})$ to encircle the pole in the standard manner and write the contour integral in terms of a principle part and associated residue, see Fig. 2. Such a continuation scheme gives rise to branches in the $\omega$ plane for $I(\omega, k, \hat{k})$ as shown in Fig. 3.

This analytic continuation of $(93)$ to $\operatorname{Im}(\omega)<0$ acquires the residue

$$
\begin{aligned}
& R(\omega, k, \hat{k})=\frac{|k-\hat{k}|}{k|k|} \frac{\partial \hat{E}}{\partial \omega}\left(\frac{\omega \hat{k}}{k}, \hat{k}\right) \hat{h}\left(k-\hat{k}, \frac{s_{k} s_{k-\hat{k}} \omega}{\sqrt{k^{2}-\omega^{2}}}\right) \\
& -\frac{k}{\left(k^{2}-\omega^{2}\right)^{3 / 2}} \hat{E}\left(\frac{\omega \hat{k}}{k}, \hat{k}\right) \hat{h}_{v^{1}}\left(k-\hat{k}, \frac{s_{k} s_{k-\hat{k}} \omega}{\sqrt{k^{2}-\omega^{2}}}\right),
\end{aligned}
$$

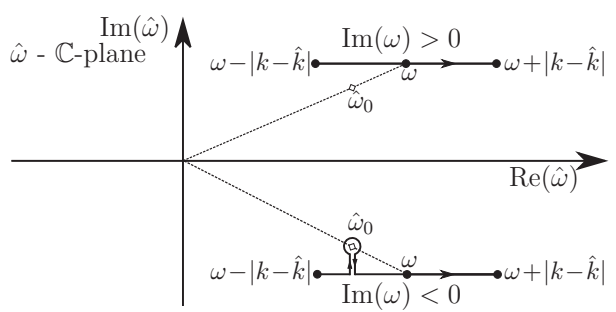

FIG. 2. The upper contour denotes $S(\omega, k, \hat{k})$ when $\operatorname{Im}(\omega)>0$ for real $k, \hat{k}$. The lower contour of integration is used when $\operatorname{Im}(\omega)<0$ for real $k, \hat{k}$. 


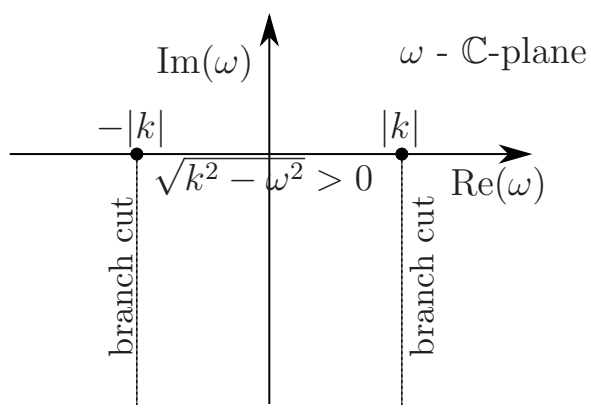

FIG. 3. Branch cuts in $\omega$ for $I(\omega, k, \hat{k})$.

where $\hat{h}_{v^{1}}\left(k, v^{1}\right)=\frac{\partial \hat{h}}{\partial v^{1}}\left(k, v^{1}\right), s_{k}=k /|k|$, and $s_{k-\hat{k}}=(k-\hat{k}) /|k-\hat{k}|$. In the case when $\operatorname{Im}(\omega)=0$, the principle value of (93) is taken together with residue $\frac{1}{2} R(\omega, k, \hat{k})$. Equation (92) then gives

$$
\begin{aligned}
& \hat{E}(\omega, k)=\frac{k}{2 \pi} \int_{-\infty}^{\infty} I(\omega, k, \hat{k}) d \hat{k} \quad \text { if } \operatorname{Im}(\omega)>0 \quad \text { or }|\operatorname{Re}(\omega)|>|k|, \\
& \hat{E}(\omega, k)=\frac{k}{2 \pi} \int_{-\infty}^{\infty} I(\omega, k, \hat{k}) d \hat{k}-i k \int_{-\infty}^{\infty} R(\omega, k, \hat{k}) d \hat{k} \\
& \hat{E}(\omega, k)=\frac{k}{2 \pi} \int_{-\infty}^{\infty} \mathcal{P} I(\omega, k, \hat{k}) d \hat{k}-\frac{i k}{2} \int_{-\infty}^{\infty} R(\omega, k, \hat{k}) d \hat{k} \\
& \text { if } \quad \operatorname{Im}(\omega)<0 \quad \text { and }|\operatorname{Re}(\omega)| \leq|k|,
\end{aligned}
$$

where $\mathcal{P} I(\omega, k, \hat{k})$ in (94) refers to the principle part of (93) when $\operatorname{Im}(\omega)=0$ and $|\operatorname{Re}(\omega)|<|k|$ and hence the pole at $\hat{\omega}_{0}$ lies on the contour $S(\omega, k, \hat{k})$. Thus in each domain above, the perturbation $\hat{E}(\omega, k)$ must be determined by solving a nonstandard integral equation.

\section{CONCLUSIONS}

In this article a classical covariant description of electromagnetic interactions in continuous matter in an arbitrary background gravitational field has been formulated in terms of a polarization 2 -form that enters into the macroscopic Maxwell equations. Linear dispersive constitutive relations arise when this 2-form is expressed as an affine functional of the Maxwell 2-form with the aid of a 2-point susceptibility kernel. We have explored the constraints on this kernel imposed by causality requirements, spacetime Killing symmetries and local gauge freedoms. The formalism has been applied to an analysis of constitutive models for waves in collisionless plasmas. In particular a formula for the linear susceptibility of a fully ionized inhomogeneous unbounded nonstationary collisionless plasma to a perturbation in the presence of gravity has been given in terms of maps describing the dynamics of the plasma. This formula has been elucidated by reference to both homogeneous and inhomogeneous perturbations in Minkowski spacetime. In the former case one recovers the standard Landau dispersion relation when perturbing Langmuir modes. In the latter case we have described a generalized damping mechanism for such modes that may arise when the unperturbed state is both inhomogeneous and nonstationary. Such a mechanism arises from the analytic continuation of an integral equation that replaces the Landau dispersion relation.

It is concluded that the use of a covariant 2-point affine susceptibility kernel in describing the electromagnetic response of dispersive media offers a modeling tool that naturally generalizes the use of permittivity and permeability tensors used to model electromagnetic interactions in nonrelativistic media. The formulation in terms of an arbitrary background spacetime metric offers 
potential applications in a number of astrophysical contexts involving electromagnetic fields in inhomogeneous or nonstationary plasmas.

\section{ACKNOWLEDGMENTS}

The authors are grateful to support from Engineering and Physical Sciences Research Consul (U.K.) (EPSRC)(EP/E001831/1) and the Cockcroft Institute (STFC ST/G008248/1).

\section{APPENDIX: PROOFS OF RESULTS USED USED IN THE TEXT}

Lemma 2: Local representation of $\oint_{\pi_{\mathcal{N}}} \alpha$ in (4) from the implicit definition in Eq. (3).

Proof: On a fibered manifold $\mathcal{N}$ of dimension $n+r$ with projection $\pi_{\mathcal{N}}: \mathcal{N} \rightarrow N$ over a manifold $N$ of dimension $n$. Thus at each point $\sigma \in N$ one has the fiber $\mathcal{N}_{\sigma}=\pi_{\mathcal{N}}^{-1}\{\sigma\}$ $=\left\{\left(\sigma^{\prime}, \varsigma\right) \in \mathcal{N} \mid \pi_{\mathcal{N}}\left(\sigma^{\prime}, \varsigma\right)=\sigma\right\}$ so $\operatorname{dim}\left(\mathcal{N}_{\sigma}\right)=r$ is the fiber dimension. Let $\left(\sigma^{1}, \ldots, \sigma^{n}\right)$ and $\left(\sigma^{1}, \ldots, \sigma^{n}, \varsigma^{1} \ldots \varsigma^{r}\right)$ be local coordinates for patches on $N$ and $\mathcal{N}$, respectively.

Consider first the case when $\alpha \in \Gamma \Lambda^{p+r} \mathcal{N}$ consists of a single component $\alpha_{I}(\sigma, \varsigma) d \sigma^{I} \wedge$ $d \varsigma^{1 \ldots r}$ with no sum on $I$. Hence explicit summation will be used in this particular proof. Set $\hat{I}=\{1, \ldots, n\} \backslash I$ so that $d \sigma^{\hat{I}} \wedge d \sigma^{I}= \pm d \sigma^{1 \ldots n}$ and let $\beta=\sum_{J} \beta_{J}(\sigma) d \sigma^{J}$ then $\beta \wedge d \sigma^{I}$ $= \pm \beta_{\hat{I}} \alpha_{I} d \sigma^{1 \ldots n}$ so that

$$
\begin{aligned}
\sum_{J} \int_{(\sigma, \zeta) \in \mathcal{N}} \pi_{\mathcal{N}}^{\star}\left(\beta_{J}(\sigma) d \sigma^{J}\right) \wedge \alpha_{I}(\sigma, \varsigma) d \sigma^{I} \wedge d \varsigma^{1 \ldots r} \\
=\sum_{J} \int_{(\sigma, \zeta) \in \mathcal{N}} \beta_{J}(\sigma) d \sigma^{J} \wedge \alpha_{I}(\sigma, \varsigma) d \sigma^{I} \wedge d \varsigma^{1 \ldots r} \\
=\sum_{J} \int_{(\sigma, \zeta) \in \mathcal{N}} \beta_{J}(\sigma) d \sigma^{J} \wedge d \sigma^{I} \wedge \alpha_{I}(\sigma, \varsigma) d \varsigma^{1 \ldots r} \\
=\int_{(\sigma, \varsigma) \in \mathcal{N}} \beta_{\hat{I}}(\sigma) d \sigma^{\hat{I}} \wedge d \sigma^{I} \wedge \alpha_{I}(\sigma, \varsigma) d \varsigma^{1 \ldots r} \\
=\int_{\sigma \in N} \beta_{\hat{I}}(\sigma) d \sigma^{\hat{I}} \wedge d \sigma^{I} \int_{\mathcal{N}_{\sigma}} \alpha_{I}(\sigma, \varsigma) d \varsigma^{1 \ldots r} \\
=\sum_{J} \int_{\sigma \in N} \beta_{J}(\sigma) d \sigma^{J} \wedge d \sigma^{I} \int_{\mathcal{N}_{\sigma}} \alpha_{I}(\sigma, \varsigma) d \varsigma^{1 \ldots r} \\
=\int_{\sigma \in N} \beta \wedge d \sigma^{I} \int_{\mathcal{N}_{\sigma}} \alpha_{I}(\sigma, \varsigma) d \varsigma^{1 \ldots r} .
\end{aligned}
$$

Thus by linearity

$$
\int_{\mathcal{N}} \pi_{\mathcal{N}}^{\star}(\beta) \wedge \alpha=\sum_{I} \int_{\sigma \in N} \beta \wedge d \sigma^{I} \int_{\mathcal{N}_{\sigma}} \alpha_{I}(\sigma, \varsigma) d \varsigma^{1 \ldots r}
$$

where $\alpha=\sum_{I} \alpha_{I}(\sigma, \varsigma) d \sigma^{I} \wedge d \varsigma^{1 \ldots r}$. If (4) holds then for $\alpha=\sum_{I} \alpha_{I}(\sigma, \varsigma) d \sigma^{I} \wedge d \varsigma^{1 \ldots r}$,

$$
\begin{aligned}
\int_{N} \beta \wedge \mathcal{\psi}_{\pi_{\mathcal{N}}} \alpha & =\left.\sum_{I} \int_{N} \beta \wedge d \sigma^{I} \int_{\varsigma \in \mathcal{N}_{\sigma}} i_{I}^{(\sigma)} \alpha\right|_{(\sigma, \zeta)}=\sum_{I} \int_{N} \beta \wedge d \sigma^{I} \int_{\varsigma \in \mathcal{N}_{\sigma}} \alpha_{I} d \varsigma^{1 \ldots r} \\
& =\int_{\mathcal{N}} \pi_{\mathcal{N}}^{\star}(\beta) \wedge \alpha .
\end{aligned}
$$


Hence (3). Conversely if (3) holds for $\alpha=\sum_{I} \alpha_{I}(\sigma, \varsigma) d \sigma^{I} \wedge d \varsigma^{1 \ldots r}$ then from (A1)

$$
\int_{N} \beta \wedge \oint_{\pi_{\mathcal{N}}} \alpha=\int_{\mathcal{N}} \pi_{\mathcal{N}}^{\star}(\beta) \wedge \alpha=\left.\sum_{I} \int_{N} \beta \wedge d \sigma^{I} \int_{\zeta \in \mathcal{N}_{\sigma}} i_{I}^{(\sigma)} \alpha\right|_{(\sigma, \zeta)} .
$$

Since this is true for all $\beta$ then (4) holds.

If $\alpha$ does not contain the factor $\varsigma^{1 \ldots r}$, i.e., $\alpha=\alpha_{I K}(\sigma, \varsigma) d \sigma^{I} \wedge d \varsigma^{K}$, where $K \neq\{1, \ldots, r\}$ then the right hand side of (3) becomes

$$
\int_{\mathcal{N}} \pi_{\mathcal{N}}^{\star}(\beta) \wedge \alpha=\sum_{J} \int_{\mathcal{N}} \beta_{J} \alpha_{I K}(\sigma, \varsigma) d \sigma^{J} \wedge d \sigma^{I} \wedge d \varsigma^{K}=0
$$

and the right hand side of (4) becomes

$$
\sum_{I} d \sigma^{I} \int_{\varsigma \in \mathcal{N}_{\sigma}} \alpha_{I K}(\sigma, \varsigma) d \varsigma^{K}=0
$$

Thus by linearity (3) and (4) are equivalent for all $\alpha$.

Lemma 3: Verification of Eq. (5)

$$
\left.\left(d \oint_{\pi_{\mathcal{N}}} \alpha\right)\right|_{\sigma}=\left.\left(\oint_{\pi_{\mathcal{N}}} d \alpha\right)\right|_{\sigma}
$$

Proof Let $\operatorname{deg}(\alpha)=p+r, \operatorname{deg}(\beta)=n-p-1$ and $\partial N$ and $\partial \mathcal{N}$ be the boundaries of $N$ and $\mathcal{N}$. Since $\sigma \notin \partial N$ one may choose $\beta$ to have support away from $\partial N$ thus

$$
\int_{\partial N} \beta \wedge\left(\oint_{\pi_{\mathcal{N}}} \alpha\right)=0
$$

and since $\alpha$ has support away from $\partial \mathcal{N}$ then

$$
\int_{\partial \mathcal{N}} \pi_{\mathcal{N}}^{\star} \beta \wedge \alpha=0
$$

It follows that

$$
\begin{aligned}
\int_{N} \beta \wedge\left(\mathcal{f}_{\pi_{\mathcal{N}}} d \alpha\right) & =\int_{\mathcal{N}} \pi_{\mathcal{N}}^{\star}(\beta) \wedge d \alpha \\
& =(-1)^{n-p-1} \int_{\mathcal{N}} d\left(\pi_{\mathcal{N}}^{\star}(\beta) \wedge \alpha\right)+(-1)^{n-p} \int_{\mathcal{N}} d \pi_{\mathcal{N}}^{\star}(\beta) \wedge \alpha \\
& =(-1)^{n-p-1} \int_{\partial \mathcal{N}} \pi_{\mathcal{N}}^{\star}(\beta) \wedge \alpha+(-1)^{n-p} \int_{\mathcal{N}} \pi_{\mathcal{N}}^{\star}(d \beta) \wedge \alpha \\
& =(-1)^{n-p} \int_{N} d \beta \wedge\left(\oint_{\pi_{\mathcal{N}}} \alpha\right) \\
& =(-1)^{n-p} \int_{N} d\left(\beta \wedge\left(\oint_{\pi_{\mathcal{N}}} \alpha\right)\right)+\int_{N} \beta \wedge d\left(\oint_{\pi_{\mathcal{N}}} \alpha\right) \\
& =(-1)^{n-p} \int_{\partial N} \beta \wedge\left(\oint_{\pi_{\mathcal{N}}} \alpha\right)+\int_{N} \beta \wedge d\left(\oint_{\pi_{\mathcal{N}}} \alpha\right) \\
& =\int_{N} \beta \wedge d\left(\oint_{\pi_{\mathcal{N}}} \alpha\right) .
\end{aligned}
$$


Lemma 4: Proof of

$$
\oint_{p_{X}} \star_{X} \alpha=\star \oint_{p_{X}} \alpha
$$

Proof: The only nontrivial $\alpha \in \Gamma \Lambda\left(M_{X} \times M_{Y}\right)$ in (A2) can be written $\alpha=\alpha_{I} d x^{I} \wedge d y^{0123}$. Then

$\oint_{p_{X}}^{\star_{X}}\left(\alpha_{I} d x^{I} \wedge d y^{0123}\right)=\oint_{p_{X}} \alpha_{I}\left(\star d x^{I}\right) \wedge d y^{0123}=\star d x^{I} \int_{M_{X}} \alpha_{I} d y^{0123}=\star \oint_{p_{X}} \alpha_{I} d x^{I} \wedge d y^{0123}$.

Lemma 5: $\Pi$ is causal on $M_{X}^{+}$if and only if

- $Z$ is causal on $M_{X}{ }^{+}$,

- $\left.\left(d_{Y} \chi\right)\right|_{(x, y)}=0$ for all $(x, y) \in M_{X}^{+} \times M_{Y}^{+}$such that $y \notin J^{-}(x)$, and

- $\left.\iota_{\Sigma_{M_{Y}}}(\chi)\right|_{(x, y)}=0$ for all $(x, y) \in M_{X}^{+} \times \Sigma_{M_{Y}}$ such that $y \notin J^{-}(x)$, where $\iota_{\Sigma_{M_{Y}}}: M_{X}^{+} \times \Sigma_{M_{Y}} \hookrightarrow M_{X}^{+} \times M_{Y}^{+}$is the natural embedding.

Proof: If $\hat{\iota}_{\Sigma_{M_{Y}}}: \Sigma_{M_{Y}} \hookrightarrow M_{Y}^{+}$is the natural embedding then $\left.i_{a b}^{(x)} \iota_{\Sigma_{M_{Y}}}^{\star} \chi\right|_{(x, y)}=\left.\hat{\iota}_{\Sigma_{M_{Y}}^{\star}} i_{a b}^{(x)} \chi\right|_{(x, y)}$ and

$$
\begin{aligned}
& \int_{y \in M_{Y}^{+}} i_{a b}^{(x)}\left(\chi \mid \wedge p_{Y}^{\star}\left(\left.d A\right|_{y}\right)\right) \\
= & \left.\int_{y \in M_{Y}^{+}} i_{a b}^{(x)}\left(\chi \wedge d_{Y}\left(p_{Y}^{\star} A\right)\right)\right|_{(x, y)} \\
= & \left.\int_{y \in M_{Y}^{+}} i_{a b}^{(x)} d_{Y}\left(\left.\chi \wedge p_{Y}^{\star} A\right|_{y}\right)\right|_{(x, y)}-\left.\int_{y \in M_{Y}^{+}} i_{a b}^{(x)}\left(\left.d_{Y} \chi \wedge p_{Y}^{\star} A\right|_{y}\right)\right|_{(x, y)} \\
= & \left.\int_{y \in M_{Y}^{+}} d_{Y}\left(\left.i_{a b}^{(x)} \chi \wedge p_{Y}^{\star} A\right|_{y}\right)\right|_{(x, y)}-\left.\int_{y \in M_{Y}^{+}} i_{a b}^{(x)}\left(\left.d_{Y} \chi \wedge p_{Y}^{\star} A\right|_{y}\right)\right|_{(x, y)} \\
= & \left.\int_{y \in \Sigma_{M_{Y}}} \hat{\iota}_{\Sigma_{M_{Y}}} i_{a b}^{(x)}\left(\left.\chi \wedge p_{Y}^{\star} A\right|_{y}\right)\right|_{(x, y)}-\left.\int_{y \in M_{Y}^{+}} i_{a b}^{(x)}\left(\left.d_{Y} \chi \wedge p_{Y}^{\star} A\right|_{y}\right)\right|_{(x, y)} \\
= & \left.\int_{y \in \Sigma_{M_{Y} \backslash J^{-}(x)}} i_{a b}^{(x)} \iota_{\Sigma_{M_{Y}}}^{\star}\left(\left.\chi \wedge p_{Y}^{\star} A\right|_{y}\right)\right|_{(x, y)}+\left.\int_{y \in \Sigma_{M_{Y}} \cap J^{-}(x)} i_{a b}^{(x)} \iota_{\Sigma_{M_{Y}}}\left(\left.\chi \wedge p_{Y}^{\star} A\right|_{y}\right)\right|_{(x, y)} \\
& -\left.\int_{y \in M_{Y}^{+} \backslash J^{-}(x)} i_{a b}^{(x)}\left(\left.d_{Y} \chi \wedge p_{Y}^{\star} A\right|_{y}\right)\right|_{(x, y)}-\left.\int_{y \in M_{Y}^{+} \cap J^{-}(x)} i_{a b}^{(x)}\left(\left.d_{Y} \chi \wedge p_{Y}^{\star} A\right|_{y}\right)\right|_{(x, y)} .
\end{aligned}
$$

First one argues that (A3) implies that $\Pi$ is causal on $M_{X}^{+}$. Given $x \in M_{X}^{+}$and $F_{1}, F_{2} \in \Gamma \Lambda^{2} M_{Y}^{+}$ such that $\left.F_{1}\right|_{y}=\left.F_{2}\right|_{y}=0$ for $y \in J^{-}(y)$, set $F=F_{1}-F_{2}$ so that $F=0$ on $J^{-}(x)$. Since $M_{Y}^{+}$ is topologically trivial $F$ is exact, $F=d \hat{A}$, and hence $d \hat{A}=0$ on $J^{-}(x)$. Then since $J^{-}(x)$ is topologically trivial there exists $f \in \Gamma \Lambda^{0} M_{Y}^{+}$such that $\hat{A}=d f$ on $J^{-}(x)$. Thus one can choose a gauge $A=\hat{A}-d f$ so that $A=0$ on $J^{-}(x)$. Given $\zeta$ such that $\left.\zeta\right|_{y}=0$ for $y \in J^{-}(x) \cap \Sigma_{M_{Y}}$ then $\left.Z[\zeta]\right|_{x}=0$ since $Z$ is causal. Thus from (A4)

$$
\begin{aligned}
\Pi[F, \zeta]_{a b}(x)= & \int_{y \in M_{Y}^{+}} i_{a b}^{(x)}\left(\chi \mid \wedge p_{Y}^{\star}\left(\left.d A\right|_{y}\right)\right) \\
= & \left.\int_{y \in \Sigma_{M_{Y} \backslash J^{-}(x)}} i_{a b}^{(x)} \iota_{\Sigma_{M_{Y}}}\left(\left.\chi \wedge p_{Y}^{\star} A\right|_{y}\right)\right|_{(x, y)}+\left.\int_{y \in \Sigma_{M_{Y}} \cap J^{-}(x)} i_{a b}^{(x)} \iota_{\Sigma_{M_{Y}}}\left(\left.\chi \wedge p_{Y}^{\star} A\right|_{y}\right)\right|_{(x, y)} \\
& -\left.\int_{y \in M_{Y}^{+} \backslash J^{-}(x)} i_{a b}^{(x)}\left(\left.d_{Y} \chi \wedge p_{Y}^{\star} A\right|_{y}\right)\right|_{(x, y)}-\left.\int_{y \in M_{Y}^{+} \cap J^{-}(x)} i_{a b}^{(x)}\left(\left.d_{Y} \chi \wedge p_{Y}^{\star} A\right|_{y}\right)\right|_{(x, y)}=0,
\end{aligned}
$$

since $\left.\iota_{\Sigma_{M_{Y}}^{\star}} \chi\right|_{(x, y)}=0$ for $y \in \Sigma_{M_{Y}} \backslash J^{-}(x),\left.A\right|_{y}=0$ for $y \in J^{-}(x)$, and $d_{Y} \chi=0$ for $y \in M_{Y}^{+} \backslash J^{-}(x)$. 
Conversely if $\Pi$ is causal on $M_{X}^{+}$then setting $F=0$ in (6) shows that $Z$ must be causal on $M_{X}^{+}$. Then setting $\zeta=0$ then for all $A$ such that $A=0$ on $J^{-}(x)$ (A4) yields

$$
\begin{aligned}
0 & =\Pi[F, \zeta]_{a b}(x) \\
& =\left.\int_{y \in \Sigma_{M_{Y} \backslash J^{-}(x)}} i_{a b}^{(x)} \iota_{\Sigma_{M_{Y}}^{\star}}\left(\left.\chi(x, y) \wedge p_{Y}^{\star} A\right|_{y}\right)\right|_{(x, y)}-\left.\int_{y \in M_{Y}^{+} \backslash J^{-}(x)} i_{a b}^{(x)}\left(\left.d_{Y} \chi_{(x, y)} \wedge p_{Y}^{\star} A\right|_{y}\right)\right|_{(x, y)} .
\end{aligned}
$$

The four-dimensional domain $M_{Y}^{+} \backslash J^{-}(x)$ denotes points outside the backward light-cone of $x$, while the three-dimensional domain $\Sigma_{M_{Y}} \backslash J^{-}(x)$ denotes the points on $\Sigma_{M_{Y}}$ that are not causally connected to $x$. Choosing such an $A$ to have support about a small neighborhood of $y \in M_{Y}^{+} \backslash J^{-}(x) \backslash \Sigma_{M_{Y}}$ results in the first term of (A5) being zero and thus $\left.\left(d_{Y} \chi\right)\right|_{(x, y)}=0$. Likewise setting $A$ to have support

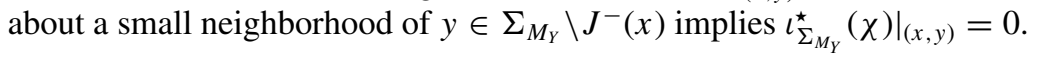

One can now prove Lemma 1 in Sec. III B.

Proof of Lemma 1: Given $\sigma \in N$, with $V$ nonvanishing there exists a coordinate system $\left(\sigma^{1}, \ldots, \sigma^{n}\right)$ on $N$ adapted to $V$ so that $V=\frac{\partial}{\partial \sigma^{\mathrm{I}}}$ and the image of the curve $\gamma_{\sigma}:\left[\tau_{0}(\sigma), 0\right] \rightarrow N$ is contained in the coordinate patch. Write $\beta=\beta_{I} d \sigma^{I}$ then since $i_{V} \beta=0$ the sum is over $I \in\{2, \ldots, n\}$. With $\sigma^{1}$ distinguished write $\beta_{I}(\sigma)=\beta_{I}\left(\sigma^{1}, \underline{\sigma}\right)$, where $\underline{\sigma}=\left(\sigma^{2}, \ldots, \sigma^{n}\right)$. Also since $i_{V} \beta=0,\left.\beta\right|_{\left(\sigma^{1}, \underline{\sigma}\right)}=\beta_{I}\left(\sigma^{1}, \underline{\sigma}\right) d \underline{\sigma}^{I}$. Likewise since $i_{V} \zeta=0$ one has $\left.\zeta\right|_{\sigma_{0}}=\zeta_{I}\left(\sigma_{0}\right) d \underline{\sigma}^{I}$.

Solving for the integral curves of $V$ gives $\phi_{N}\left(\tau, \sigma^{1}, \underline{\sigma}\right)=\left(\tau+\sigma^{1}, \underline{\sigma}\right)$,

$$
\left.\phi_{N}^{\star}(\beta)\right|_{\left(\tau, \sigma^{1}, \underline{\sigma}\right)}=\beta_{I}\left(\tau+\sigma^{1}, \underline{\sigma}\right) d \underline{\sigma}^{I},
$$

and one may write $\tau_{0}\left(\sigma^{1}, \underline{\sigma}\right)=\tau_{0}(\underline{\sigma})-\sigma^{1}$, giving

$$
\left.\varphi_{N}(\zeta)\right|_{\left(\sigma^{1}, \underline{\sigma}\right)}=\phi_{N \tau_{0}\left(\sigma^{1}, \underline{\sigma}\right)}\left(\left.\zeta\right|_{\tau_{0}(\underline{\sigma})}\right)=\zeta_{I}\left(\tau_{0}(\underline{\sigma}), \underline{\sigma}\right) d \underline{\sigma}^{I} .
$$

Thus,

$$
\begin{aligned}
\left.\xi\right|_{\left(\sigma^{1}, \underline{\sigma}\right)} & =\oint_{\varpi_{N}} \phi_{N}^{\star}(\beta) \wedge d \tau+\varphi_{N}(\zeta) \\
& =\left(\int_{\tau=\tau_{0}(\underline{\sigma})-\sigma^{1}}^{0} \beta_{I}\left(\sigma^{1}+\tau, \underline{\sigma}\right) d \tau+\zeta_{I}\left(\tau_{0}(\underline{\sigma}), \underline{\sigma}\right)\right) d \underline{\sigma}^{I} .
\end{aligned}
$$

Hence $i_{V} \xi=0$ and one may write $\left.\xi\right|_{\left(\sigma^{1}, \underline{\sigma}\right)}=\xi_{I}\left(\sigma^{1}, \underline{\sigma}\right) d \underline{\sigma}^{I}$. Now

$$
\begin{aligned}
\xi_{I}\left(\sigma^{1}, \underline{\sigma}\right) & =\int_{\tau=\tau_{0}(\underline{\sigma})-\sigma^{1}}^{0} \beta_{I}\left(\sigma^{1}+\tau, \underline{\sigma}\right) d \tau+\zeta_{I}\left(\tau_{0}(\underline{\sigma}), \underline{\sigma}\right) \\
& =\int_{\tau=\tau_{0}(\underline{\sigma})}^{\sigma^{1}} \beta_{I}\left(\tau^{\prime}, \underline{\sigma}\right) d \tau^{\prime}+\zeta_{I}\left(\tau_{0}(\underline{\sigma}), \underline{\sigma}\right),
\end{aligned}
$$

where $\tau^{\prime}=\tau+\sigma^{1}$ and

$$
\begin{aligned}
\left.i_{V} d \xi\right|_{\left(\sigma^{1}, \underline{\sigma}\right)} & =i_{\frac{\partial}{\partial \sigma^{I}}} d\left(\xi_{I}\left(\sigma^{1}, \underline{\sigma}\right) d \underline{\sigma}^{I}\right)=i_{\frac{\partial}{\partial \sigma^{I}}}\left(d \xi_{I}\left(\sigma^{1}, \underline{\sigma}\right) \wedge d \underline{\sigma}^{I}\right)=\frac{\partial \xi_{I}\left(\sigma^{1}, \underline{\sigma}\right)}{\partial \sigma^{1}} d \underline{\sigma}^{I} \\
& =\frac{\partial}{\partial \sigma^{1}}\left(\int_{\tau=\tau_{0}(\underline{\sigma})}^{\sigma^{1}} \beta_{I}\left(\tau^{\prime}, \underline{\sigma}\right) d \tau^{\prime}+\zeta_{I}\left(\tau_{0}(\underline{\sigma}), \underline{\sigma}\right)\right) d \underline{\sigma}^{I}=\beta_{I}\left(\sigma^{1}, \underline{\sigma}\right) d \underline{\sigma}^{I}=\left.\beta\right|_{\left(\sigma^{1}, \underline{\sigma}\right)} .
\end{aligned}
$$

Since $\sigma^{1}=0$ on $\Sigma_{N}$

$$
\left.\xi\right|_{(0, \underline{\sigma})}=\xi_{I}\left(\tau_{0}(0, \underline{\sigma}), \underline{\sigma}\right) d \underline{\sigma}^{I}=\xi_{I}\left(\tau_{0}(\underline{\sigma}), \underline{\sigma}\right) d \underline{\sigma}^{I}=\zeta_{I}\left(\tau_{0}(\underline{\sigma}), \underline{\sigma}\right) d \underline{\sigma}^{I}=\left.\zeta\right|_{(0, \underline{\sigma})},
$$

i.e., $\left.\xi\right|_{\Sigma_{N}}=\zeta$.

Lemma 6: Proof that (66) and (67) implies (61) and that (66) and (69) implies (61). 
Proof: First (67) is equivalent to (69) since given $\gamma \in \Gamma \Lambda^{2} M_{Y}^{+}$one has $\widetilde{i_{(y, u)} \gamma}=u^{a} \gamma_{a b} g^{b c} \frac{\partial}{\partial y^{c}}$ and hence $\hat{W}^{[\alpha]}(\gamma)=\frac{q^{\mid \alpha]}}{m^{[\alpha]}} \mathcal{V}_{(y, u)}\left(\widetilde{i_{(y, u)} \gamma}\right)=\frac{q^{\mid \alpha]}}{m^{[\alpha]}} u^{a} \gamma_{a b} g^{b \nu} \frac{\partial}{\partial u^{v}}$. From (67) it follows that

$$
\begin{aligned}
& \chi^{[\alpha]} \wedge p_{Y}^{\star} \gamma=\frac{1}{2} \frac{q^{[\alpha\rceil 2}}{m^{[\alpha]}} \star_{X} d y^{c d} \wedge i_{a b c d}^{(y)} \Psi^{[\alpha] \star}\left(d \tau \wedge \varpi_{Y}^{\mid \alpha\rceil \star}\left(g^{\nu a} u^{b} i_{v}^{(u)} \theta_{0}^{\lfloor\alpha]}\right)\right) \wedge p_{Y}^{\star} \gamma
\end{aligned}
$$

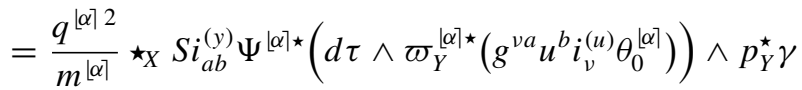

$$
\begin{aligned}
& =-\frac{q^{\lfloor\alpha\rceil 2}}{m^{\lfloor\alpha\rceil}} \star_{X} S \Psi^{\lfloor\alpha\rceil \star}\left(d \tau \wedge \varpi_{Y}^{\lfloor\alpha\rceil \star}\left(g^{\nu a} u^{b} i_{v}^{(u)} \theta_{0}^{\lfloor\alpha\rceil}\right)\right) \wedge i_{a b}^{(y)} p_{Y}^{\star} \gamma \\
& =-\frac{q^{\lfloor\alpha\rceil 2}}{m^{\lfloor\alpha\rceil}} \star_{X} S \Psi^{\lfloor\alpha\rceil \star}\left(d \tau \wedge \varpi_{Y}^{\mid \alpha\rceil \star}\left(\gamma_{a b} g^{\nu a} u^{b} i_{v}^{(u)} \theta_{0}^{\lfloor\alpha\rceil}\right)\right) \\
& =-q^{[\alpha]} \star_{X} S \Psi^{\mid \alpha\rceil \star}\left(d \tau \wedge \varpi_{Y}^{\mid \alpha\rceil \star}\left(i_{\hat{W}}[\alpha](\gamma) \theta_{0}^{\lfloor\alpha\rceil}\right)\right),
\end{aligned}
$$

i.e., (69). That (69) implies (67) follows since the above argument is true for all $\gamma$.

To prove (61) note that the domains $\mathcal{N}_{X}^{\lfloor\alpha\rceil}$ and $\mathcal{N}_{Y}^{\lfloor\alpha\rceil}$ are related via the diffeomorphism

$$
\Upsilon^{\lfloor\alpha\rceil}: \mathcal{N}_{Y}^{\lfloor\alpha\rceil} \rightarrow \mathcal{N}_{X}^{\lfloor\alpha\rceil}, \quad \Upsilon^{\lfloor\alpha\rceil}(\tau, y, u)=\left(-\tau, \dot{C}_{(y, u)}^{\lfloor\alpha]}(\tau)\right) .
$$

Thus $\Upsilon^{\lfloor\alpha\rceil \star}(d \tau)=-d \tau$ and setting $(x, v)=\dot{C}_{(y, u)}^{\mid \alpha\rceil}(\tau)$ with $\tau>0$ yields

$$
\phi^{\lfloor\alpha\rceil}\left(\Upsilon^{[\alpha]}(\tau, y, u)\right)=\phi^{\lfloor\alpha\rceil}\left(-\tau, \dot{C}_{(y, u)}^{[\alpha]}(\tau)\right)=\phi^{[\alpha]}(-\tau, x, v)=(y, u)=\varpi_{Y}^{\lfloor\alpha\rceil}(\tau, y, u)
$$

so that $\varpi_{Y}^{\lfloor\alpha\rceil}=\phi^{[\alpha]} \circ \Upsilon^{[\alpha]}$ and thus $\varpi_{Y}^{\mid \alpha\rceil \star}=\Upsilon^{\lfloor\alpha] \star} \circ \phi^{[\alpha] \star}$. Now

$$
\begin{aligned}
& \Upsilon^{\lfloor\alpha\rceil \star}\left(d \tau \wedge \phi^{[\alpha\rceil \star}\left(i_{\hat{W}^{\lfloor\alpha\rceil}\left(F_{1}\right)} \theta_{0}^{\lfloor\alpha\rceil}\right)\right) \\
& =\Upsilon^{[\alpha] \star}(d \tau) \wedge \Upsilon^{\lfloor\alpha\rceil \star} \phi^{[\alpha\rceil \star}\left(i_{\hat{W} \mid \alpha]_{\left(F_{1}\right)}} \theta_{0}^{\mid \alpha\rceil}\right)=-d \tau \wedge \varpi_{Y}^{\mid \alpha\rceil \star}\left(i_{\hat{W}^{\lfloor\alpha]}\left(F_{1}\right)} \theta_{0}^{\lfloor\alpha\rceil}\right) \text {, }
\end{aligned}
$$

hence

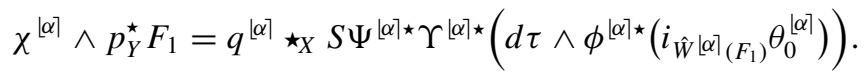

From (63)

$$
p_{X}\left(\Phi^{\mid \alpha\rceil}(\tau, y, u)\right)=p_{X}\left(C_{(y, u)}^{\mid \alpha\rceil}(\tau), y\right)=C_{(y, u)}^{\lfloor\alpha\rceil}(\tau)
$$

and from (A6)

$$
\pi_{X}\left(\varpi_{X}^{\mid \alpha\rceil}\left(\Upsilon^{\lfloor\alpha\rceil}(\tau, y, u)\right)\right)=\pi_{X}\left(\varpi_{X}^{\lfloor\alpha\rceil}\left(-\tau, \dot{C}_{(y, u)}^{\lfloor\alpha\rceil}(\tau)\right)\right)=\pi_{X}\left(\dot{C}_{(y, u)}^{\lfloor\alpha\rceil}(\tau)\right)=C_{(y, u)}^{\lfloor\alpha\rceil}(\tau) .
$$

Hence $p_{X} \circ \Phi^{\lfloor\alpha]}=\pi_{X} \circ \varpi_{X}^{[\alpha]} \circ \Upsilon^{\mid \alpha\rceil}$ and so

$$
\Phi^{[\alpha] \star} \circ p_{X}^{\star}=\Upsilon^{[\alpha] \star} \circ \varpi_{X}^{[\alpha] \star} \circ \pi_{X}^{\star} .
$$

From the definition of $S$ one has

$$
\oint_{p_{X}} S \gamma=\oint_{p_{X}} \gamma
$$

for any $\gamma \in \Gamma \Lambda^{8}\left(M_{X} \times M_{Y}\right)$. 
Since $\Psi^{\lfloor\alpha\rceil}: \mathcal{D} \rightarrow \mathcal{D}^{\prime}$ is a diffeomorphism then

$$
\int_{\mathcal{D}} \Psi^{\lfloor\alpha\rceil \star} \gamma=\int_{\mathcal{D}^{\prime}} \gamma
$$

for any $\gamma \in \Gamma \Lambda^{8}\left(\mathcal{D}^{\prime}\right)$. Likewise since $\Upsilon^{\lfloor\alpha\rceil}: \mathcal{N}_{Y}^{\lfloor\alpha\rceil} \rightarrow \mathcal{N}_{X}^{\lfloor\alpha\rceil}$ is a diffeomorphism

$$
\int_{\mathcal{N}_{Y}^{\lfloor\alpha\rceil}} \Upsilon^{\lfloor\alpha\rceil \star} \gamma=\int_{\mathcal{N}_{X}^{\lfloor\alpha\rceil}} \gamma
$$

for any $\gamma \in \Gamma \Lambda^{8}\left(\mathcal{N}_{X}^{\lfloor\alpha\rceil}\right)$.

For convenience set $\alpha^{\lfloor\alpha\rceil}=d \tau \wedge \phi^{\lfloor\alpha\rceil \star}\left(i_{\hat{W}\lfloor\alpha\rceil} F_{\left(F_{1}\right)} \theta_{0}^{\lfloor\alpha\rceil}\right) \in \Gamma \Lambda^{5} \mathcal{N}_{X}^{\lfloor\alpha\rceil}$. For fixed $x$ assume that $F_{1}$ has support in $\mathcal{D}_{x}$. Then one can choose $\beta \in \Gamma \Lambda^{2} M_{X}$ so that $p_{X}^{\star} \beta \wedge p_{Y}^{\star} F_{1}$ has support inside $\mathcal{D}$. Thus from (A7)

$$
\operatorname{supp}\left(p_{X}^{\star}(\star \beta) \wedge \Psi^{\mid \alpha\rceil \star} \Upsilon^{\mid \alpha\rceil \star} \alpha^{\lfloor\alpha\rceil}\right)=\operatorname{supp}\left(p_{X}^{\star} \beta \wedge \chi^{\lfloor\alpha\rceil} \wedge p_{Y}^{\star} F_{1}\right) \subset \mathcal{D}
$$

Now

$$
\begin{aligned}
& \int_{M_{X}} \beta \wedge \oint_{p_{X}} \chi^{\lfloor\alpha\rceil} \wedge p_{Y}^{\star} F_{1}=\int_{M_{X}} \beta \wedge \oint_{p_{X}} q^{\lfloor\alpha\rceil} \star_{X} S \Psi^{\lfloor\alpha\rceil \star} \Upsilon^{\lfloor\alpha] \star} \alpha^{\lfloor\alpha\rceil} \quad \text { from (A7) } \\
& =q^{\lfloor\alpha\rceil} \int_{M_{X}} \beta \wedge \star \int_{p_{X}} S \Psi^{\lfloor\alpha\rceil \star} \Upsilon^{\lfloor\alpha\rceil \star} \alpha^{\lfloor\alpha\rceil} \quad \text { from (A2) } \\
& =q^{\lfloor\alpha\rceil} \int_{M_{X}} \beta \wedge \star \oint_{p_{X}} \Psi^{\lfloor\alpha\rceil \star} \Upsilon^{\lfloor\alpha\rceil \star} \alpha^{\lfloor\alpha\rceil} \quad \text { from (A9) } \\
& =-q^{\lfloor\alpha]} \int_{M_{X}}(\star \beta) \wedge \oint_{p_{X}} \Psi^{\lfloor\alpha] \star} \Upsilon^{\lfloor\alpha] \star} \alpha^{[\alpha]} \\
& =-q^{\lfloor\alpha\rceil} \int_{M_{X} \times M_{Y}} p_{X}^{\star}(\star \beta) \wedge \Psi^{\lfloor\alpha\rceil \star} \Upsilon^{\lfloor\alpha\rceil \star} \alpha^{\lfloor\alpha\rceil} \quad \text { from (3) } \\
& =-q^{\lfloor\alpha\rceil} \int_{\mathcal{D}} p_{X}^{\star}(\star \beta) \wedge \Psi^{\lfloor\alpha\rceil \star} \Upsilon^{\lfloor\alpha\rceil \star} \alpha^{\lfloor\alpha\rceil} \quad \text { from (A12) } \\
& =-q^{\lfloor\alpha\rceil} \int_{\mathcal{D}} \Psi^{\lfloor\alpha\rceil \star}\left(\Phi^{\lfloor\alpha\rceil \star} p_{X}^{\star}(\star \beta) \wedge \Upsilon^{\lfloor\alpha\rceil \star} \alpha^{\lfloor\alpha\rceil}\right) \text { from (64) } \\
& =-q^{\lfloor\alpha\rceil} \int_{\mathcal{D}^{\prime}} \Phi^{\lfloor\alpha\rceil \star} p_{X}^{\star}(\star \beta) \wedge \Upsilon^{\lfloor\alpha\rceil \star} \alpha^{[\alpha\rceil} \quad \text { from (A10) } \\
& =-q^{\lfloor\alpha\rceil} \int_{\mathcal{N}_{Y}^{\lfloor\alpha\rceil}} \Phi^{\lfloor\alpha\rceil \star} p_{X}^{\star}(\star \beta) \wedge \Upsilon^{\lfloor\alpha\rceil \star} \alpha^{\lfloor\alpha\rceil} \quad \text { since } \mathcal{D}^{\prime} \subset \mathcal{N}_{Y}^{\lfloor\alpha\rceil} \\
& =-q^{\lfloor\alpha\rceil} \int_{\mathcal{N}_{Y}^{\lfloor\alpha\rceil}} \Upsilon^{\lfloor\alpha\rceil \star} \varpi_{X}^{\mid \alpha\rceil \star} \pi_{X}^{\star}(\star \beta) \wedge \Upsilon^{\lfloor\alpha\rceil \star} \alpha^{\lfloor\alpha\rceil} \text { from (A8) } \\
& =-q^{\lfloor\alpha\rceil} \int_{\mathcal{N}_{X}^{\lfloor\alpha\rceil}} \varpi_{X}^{\lfloor\alpha\rceil \star} \pi_{X}^{\star}(\star \beta) \wedge \alpha^{\lfloor\alpha\rceil} \quad \text { from (A11) } \\
& =-q^{\lfloor\alpha\rceil} \int_{\mathcal{E}_{X}} \pi_{X}^{\star}(\star \beta) \wedge \oint_{\varpi_{X}^{\lfloor\alpha\rceil}} \alpha^{\lfloor\alpha\rceil} \quad \text { from (3) } \\
& =-q^{\lfloor\alpha\rceil} \int_{M_{X}}(\star \beta) \wedge \oint_{\pi_{X}} \oint_{\omega_{X}^{\lfloor\alpha\rceil}} \alpha^{\lfloor\alpha\rceil} \quad \text { from (3) } \\
& =q^{\lfloor\alpha\rceil} \int_{M_{X}} \beta \wedge \star \oint_{\pi_{X}} \oint_{\varpi_{X}\langle\alpha\rceil} \alpha^{\lfloor\alpha\rceil} \text {. }
\end{aligned}
$$

Summing over $\lfloor\alpha\rceil$ gives

$$
\int_{M_{X}} \beta \wedge \oint_{p_{X}} \chi \wedge p_{Y}^{\star} F_{1}=\sum_{\lfloor\alpha\rceil} q^{\lfloor\alpha\rceil} \int_{M_{X}} \beta \wedge \star \oint_{\pi_{X}} \oint_{\left.\varpi_{X} \mid \alpha\right\rceil} \alpha^{\lfloor\alpha\rceil} .
$$

Since this is true for all $\beta$ with support in a neighborhood of $x$ then (61) holds at $x$. 
Lemma 7: The derivation of (72) from (67).

Proof: The derivation of (72) from (67) follows by first writing the Liouville vector field (36) as

$$
W_{0}^{\lfloor\alpha\rceil}=u^{a} \frac{\partial}{\partial y^{a}}+H^{v} \frac{\partial}{\partial u^{v}}, \quad \text { where } \quad H^{v}=-\Gamma^{v} e_{f} u^{e} u^{f}+\frac{q^{\lfloor\alpha\rceil}}{m^{\lfloor\alpha]}} F_{0 e f} g^{v e} u^{f} .
$$

Then setting $f^{\mid \alpha]}(y, u)=f_{0}^{\mid \alpha]}(y, u)+f_{1}^{\mid \alpha]}(y, u)$ it follows from (31) that

$$
\begin{aligned}
\theta_{0}^{\lfloor\alpha\rceil} & =i_{W_{0}^{\mid \alpha\rceil}}\left(f_{0}^{\lfloor\alpha\rceil} \Omega\right)=f_{0}^{\lfloor\alpha\rceil} i_{W_{0}^{\lfloor\alpha\rceil}}\left(\frac{|\operatorname{det} g|}{u_{0}} d y^{0123} \wedge d u^{123}\right) \\
& =f_{0}^{\lfloor\alpha\rceil} \frac{|\operatorname{det} g|}{u_{0}}\left(u^{c} Y_{c} \wedge d u^{123}+\frac{1}{2} H^{\mu} \epsilon_{\mu \nu \sigma} Y \wedge d u^{\nu \sigma}\right),
\end{aligned}
$$

where $Y_{a}=i_{\frac{\partial}{\partial y^{a}}} d y^{0123}$ and $Y=d y^{0123}$. Consequently

$$
\begin{gathered}
g^{\mu a} u^{b} i_{\mu}^{(u)} \theta_{0}^{\lfloor\alpha\rceil}=f_{0}^{\lfloor\alpha\rceil} \frac{|\operatorname{det} g|}{u_{0}} g^{\mu a} u^{b}\left(-\frac{1}{2} u^{c} \epsilon_{\mu \nu \sigma} Y_{c} \wedge d u^{\nu \sigma}-H^{v} \epsilon_{\mu \nu \sigma} Y \wedge d u^{\sigma}\right), \\
-d \tau \wedge g^{\mu a} u^{b} i_{\mu}^{(u)} \theta_{0}^{\lfloor\alpha\rceil}=f_{0}^{\lfloor\alpha\rceil} \frac{|\operatorname{det} g|}{u_{0}} g^{\mu a} u^{b} \epsilon_{\mu \nu \sigma}\left(\frac{u^{c}}{2} d \tau \wedge Y_{c} \wedge d u^{\nu \sigma}+H^{\nu} d \tau \wedge Y \wedge d u^{\sigma}\right) .
\end{gathered}
$$

Under the maps $\varpi_{Y}^{\lfloor\alpha\rceil}$ and $\hat{\psi}^{\lfloor\alpha\rceil \star}$ one has

$$
\varpi_{Y}^{\mid \alpha\rceil \star}\left(d y^{a}\right)=d y^{a}, \quad \varpi_{Y}^{\mid \alpha\rceil \star}\left(d u^{\mu}\right)=d u^{\mu},
$$

and

$$
\hat{\psi}^{[\alpha] \star}\left(d y^{a}\right)=d y^{a}, \quad \hat{\psi}^{[\alpha] \star}\left(d u^{\mu}\right)=\frac{\partial u^{\mu}}{\partial x^{a}} d x^{a}+\frac{\partial u^{\mu}}{\partial y^{a}} d y^{a}, \quad \hat{\psi}^{[\alpha] \star}(d \tau)=\frac{\partial \tau}{\partial x^{a}} d x^{a}+\frac{\partial \tau}{\partial y^{a}} d y^{a} .
$$

So using the projector $S$ given in (70) yields

$$
\begin{array}{r}
-S \hat{\psi}^{[\alpha] \star}\left(d \tau \wedge g^{\nu a} u^{b} i_{v}^{(u)} \theta_{0}^{[\alpha]}\right)=f_{0}^{[\alpha]} \frac{|\operatorname{det} g|}{u_{0}} g^{\mu a} u^{b} \epsilon_{\mu \nu \sigma}\left(\frac{u^{c}}{2} \frac{\partial \tau}{\partial y^{c}} \frac{\partial u^{\nu}}{\partial x^{d}} \frac{\partial u^{\sigma}}{\partial x^{e}}-\frac{u^{c}}{2} \frac{\partial \tau}{\partial x^{d}} \frac{\partial u^{\nu}}{\partial y^{c}} \frac{\partial u^{\sigma}}{\partial x^{e}}\right. \\
\left.+\frac{u^{c}}{2} \frac{\partial \tau}{\partial x^{d}} \frac{\partial u^{\nu}}{\partial x^{e}} \frac{\partial u^{\sigma}}{\partial y^{c}}+H^{\nu} \frac{\partial \tau}{\partial x^{d}} \frac{\partial u^{\sigma}}{\partial x^{e}}\right) Y \wedge d x^{d e} .
\end{array}
$$

Hence from (67)

$$
\begin{aligned}
& \chi^{\lfloor\alpha\rceil}=-\frac{q^{\mid \alpha\rceil 2}}{m^{[\alpha\rceil}} \star_{X}\left(i_{a b}^{(y)} S \hat{\psi}^{[\alpha] \star}\left(d \tau \wedge \varpi_{Y}^{\star}\left(g^{\nu a} u^{b} i_{v}^{(u)} \theta_{0}^{\mid \alpha\rceil}\right)\right)\right)
\end{aligned}
$$

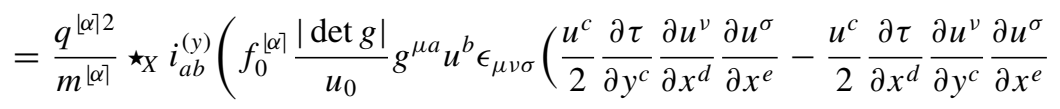

$$
\begin{aligned}
& \left.\left.+\frac{u^{c}}{2} \frac{\partial \tau}{\partial x^{d}} \frac{\partial u^{v}}{\partial x^{e}} \frac{\partial u^{\sigma}}{\partial y^{c}}+H^{v} \frac{\partial \tau}{\partial x^{d}} \frac{\partial u^{\sigma}}{\partial x^{e}}\right) Y \wedge d x^{d e}\right) \\
& =-\star_{X}\left(f _ { 0 } ^ { \lfloor \alpha ] } \frac { | \operatorname { d e t } g | } { u _ { 0 } } g ^ { \mu a } u ^ { b } \epsilon _ { \mu \nu \sigma } \epsilon _ { a b f g } \left(\frac{u^{c}}{2} \frac{\partial \tau}{\partial y^{c}} \frac{\partial u^{v}}{\partial x^{d}} \frac{\partial u^{\sigma}}{\partial x^{e}}-\frac{u^{c}}{2} \frac{\partial \tau}{\partial x^{d}} \frac{\partial u^{v}}{\partial y^{c}} \frac{\partial u^{\sigma}}{\partial x^{e}}\right.\right. \\
& \left.\left.+\frac{u^{c}}{2} \frac{\partial \tau}{\partial x^{d}} \frac{\partial u^{v}}{\partial x^{e}} \frac{\partial u^{\sigma}}{\partial y^{c}}+H^{v} \frac{\partial \tau}{\partial x^{d}} \frac{\partial u^{\sigma}}{\partial x^{e}}\right) d x^{d e} \wedge d y^{f g}\right) \\
& =\frac{q^{\mid \alpha\rceil 2}}{m^{[\alpha]}} f_{0}^{\lfloor\alpha\rceil} \frac{|\operatorname{det} g|^{3 / 2}}{2 u_{0}} g^{\mu b} u^{a} \epsilon_{\mu \nu \sigma} \epsilon_{a b f g} \epsilon^{d e h i}\left(\frac{u^{c}}{2} \frac{\partial \tau}{\partial y^{c}} \frac{\partial u^{\nu}}{\partial x^{d}} \frac{\partial u^{\sigma}}{\partial x^{e}}-\frac{u^{c}}{2} \frac{\partial \tau}{\partial x^{d}} \frac{\partial u^{\nu}}{\partial y^{c}} \frac{\partial u^{\sigma}}{\partial x^{e}}\right. \\
& \left.+\frac{u^{c}}{2} \frac{\partial \tau}{\partial x^{d}} \frac{\partial u^{v}}{\partial x^{e}} \frac{\partial u^{\sigma}}{\partial y^{c}}+H^{v} \frac{\partial \tau}{\partial x^{d}} \frac{\partial u^{\sigma}}{\partial x^{e}}\right) d x_{h i} \wedge d y^{f g} .
\end{aligned}
$$


${ }^{1}$ J. Gratus and R.W. Tucker, Prog. Electromagn. Res. 13, 145, 2010.

${ }^{2}$ Points here refer in general to events in a spacetime manifold.

${ }^{3}$ In this article the term polarization will refer to any state of the medium that gives rise to magnetization or electrical polarization in some frame.

${ }^{4}$ R. Bott and L. W. Tu, Differential Forms in Algebraic Topology (Springer, New York, 1982).

${ }^{5}$ G. De Rham, Differentiable Manifolds: Forms, Currents, Harmonic Forms (Springer-Verlag, Berlin, 1984).

${ }^{6}$ When $A$ is not compact on $M_{Y}$ invariance is modulo a boundary term.

${ }^{7}$ G. M. Wald, General Relativity (University of Chicago Press, Chicago, 1984).

${ }^{8}$ We write $y \in J^{-}(x)$ if $x$ is (timelike or lightlike) causally connected to $y$ and $x$ lies in the future of $y$.

${ }^{9}$ Note that this definition of homogeneity refers only to the electromagnetic properties of a medium.

${ }^{10}$ K. Yano and S. Ishihara, Tangent and Cotangent Bundles: Differential Geometry (Marcel Dekker, New York, 1973).

${ }^{11}$ J. Ehlers, General Relativity and Cosmology, in Proceedings of the XLVII Enrico Fermi School, edited by R. K. Sachs (Academic, New York, 1971), pp. 1-70.

${ }^{12}$ R. A. O'Sullivan and H. Derfler Phys. Rev. A 8(5), 2645, 1973. 